\title{
Weak corrections to Higgs hadroproduction in association with a top-quark pair
}

\author{
S. Frixione, ${ }^{a}$ V. Hirschi, ${ }^{b}$ D. Pagani, ${ }^{c}$ H.-S. Shao ${ }^{d}$ and M. Zaro ${ }^{e, f}$ \\ ${ }^{a}$ PH Department, TH Unit, CERN, \\ CH-1211 Geneva 23, Switzerland \\ ${ }^{b} S L A C$, National Accelerator Laboratory, \\ 2575 Sand Hill Road, Menlo Park, CA 94025-7090, U.S.A. \\ ${ }^{c}$ Centre for Cosmology, Particle Physics and Phenomenology (CP3), \\ Université Catholique de Louvain, \\ B-1348 Louvain-la-Neuve, Belgium \\ ${ }^{d}$ Department of Physics and State Key Laboratory of Nuclear Physics and Technology, \\ Peking University, \\ Beijing 100871, China \\ e Sorbonne Universités, UPMC Univ. Paris 06, UMR 7589, LPTHE, \\ F-75005, Paris, France \\ ${ }^{f}$ CNRS, UMR 7589, LPTHE, \\ F-75005, Paris, France \\ E-mail: stefano.frixione@cern.ch, vahirsch@slac.stanford.edu, \\ davide.pagani@uclouvain.be, huasheng.shao@cern.ch, \\ zaro@lpthe.jussieu.fr
}

ABstRACT: We present the calculation of the next-to-leading contribution of order $\alpha_{S}^{2} \alpha^{2}$ to the production of a Standard Model Higgs boson in association with a top-quark pair at hadron colliders. All effects of weak and QCD origin are included, whereas those of QED origin are ignored. We work in the MADGRAPH5_AMC@NLO framework, and discuss sample phenomenological applications at a 8, 13, and $100 \mathrm{TeV} p p$ collider, including the effects of the dominant next-to-leading QCD corrections of order $\alpha_{S}^{3} \alpha$.

Keywords: NLO Computations, Hadronic Colliders

ARXiv EPRINT: 1407.0823 


\section{Contents}

1 Introduction 1

2 Organisation of the calculation 3

2.1 Calculation of the $\mathcal{O}\left(\alpha_{s}^{2} \alpha^{2}\right)$ contribution with MAdGraph5_AMC@NLO 8

$\begin{array}{llr}3 & \text { Results } & 11\end{array}$

$\begin{array}{ll}3.1 & \text { Inclusive rates } \\ & 12\end{array}$

$\begin{array}{lll}3.2 & \text { Differential distributions } & 17\end{array}$

4 Conclusions $\quad 19$

\section{Introduction}

After the discovery of a new particle with a mass of $125 \mathrm{GeV}$ at the LHC [1, 2], the determination of its physical properties has become one of the main priorities in high-energy particle physics. The recent results of the ATLAS and CMS collaborations strongly suggest that this particle is the Higgs scalar boson emerging from the Brout-Englert-Higgs mechanism in the Standard Model (SM) [3-5]. In particular, the analyses of the distributions of its decay products point to a dominantly CP-even scalar [6, 7], and the fitted values for its couplings are compatible with those predicted by the SM $[8,9]$. However, the precision of the current measurements still leaves room for Beyond the Standard Model (BSM) scenarios. Thus, more accurate measurements, and their theoretical-result counterparts with matching precision, are necessary to fully understand the nature of this new particle.

In this context, an accurate determination of the $t \bar{t} H$ coupling $\lambda_{t \bar{t} H}$ is of great interest; among other things, it might also help shed light on the possible interplay of the Higgs boson and the top quark in the Electroweak Symmetry Breaking (EWSB) mechanism. To this purpose, the associated production of a Higgs boson and a top-quark pair at the LHC $(p p \rightarrow t \bar{t} H)$ offers an unique opportunity, since its cross section is directly proportional to $\lambda_{t \bar{t} H}^{2}$ at the leading order (LO). While a direct measurement of this production mode has not been achieved so far, mostly because of its small cross section and large background contamination, several searches have already been published by ATLAS and CMS [10-16], which use a variety of decay channels.

As is the case for all processes involving the Higgs, the effects of the radiative corrections to $t \bar{t} H$ production must be taken into account in order to achieve a realistic phenomenological description. Next-to-leading order (NLO) QCD effects, which were calculated more than ten years ago [17-20], increase the total cross section by a factor of about 1.3 (at a $13 \mathrm{TeV}$ LHC). Moreover, they significantly diminish the dependence of the cross section on the factorization and renormalization scales. More recently, in refs. [21, 22] 
NLO QCD corrections have also been matched to parton showers, and the differences with respect to fixed-order results are generally found to be small for inclusive and infraredinsensitive observables. Computations with the same level of perturbative accuracy have also been performed for the dominant background process to $t \bar{t} H$ production at the LHC, namely the production of a top-quark pair in association with a bottom-quark pair, without [23-25] or with $[26,27]$ parton-shower matching.

Besides QCD, electroweak (EW) effects might also lead to significant modifications of the LO predictions, particularly for differential distributions. So far, EW NLO corrections have been calculated for all of the other main Higgs production channels: gluon fusion [2831], vector-boson fusion [32, 33] and $V H$ associated production [34]. For the case of $t \bar{t} H$, they are currently not known. The purpose of this work is to amend this situation, and to present the first calculation of such corrections; similarly to what has been done as a first step in the case of $t \bar{t}$ hadroproduction [35-39], we do not include in our results effects of QED origin (dealt with in later papers [40-43] for $t \bar{t}$ ). Our computations are performed in the automated MADGRAPH5_AMC@NLO framework [44].

The motivation for separating weak and QED corrections to the $p p \rightarrow t \bar{t} H$ cross section is twofold. Firstly, it is only weak corrections which can induce effects whose size may be of the same order as the QCD ones in those regions of the phase space associated with large invariants, owing to the possible presence of Sudakov logarithms (see e.g. refs. [4548]), which compensate the stronger suppression of $\alpha$ w.r.t. that of $\alpha_{S}$. Secondly, weak corrections spoil the trivial dependence of LO and NLO QCD cross sections on $\lambda_{t \bar{t} H}$. This is because they also depend on the couplings of the Higgs to the $W$ and $Z$ bosons, and on the Higgs self-coupling, while QED corrections do not involve any of these additional couplings. Thus, if one wants to assess possible contaminations due to higher-order effects in the extraction of $\lambda_{t \bar{t} H}$, one may start by focusing on weak-only corrections.

From a technical viewpoint, by excluding QED corrections one also simplifies the structure of the calculation, and in particular that relevant to the subtraction of the infrared singularities. We note, however, that such a simplification is not particularly significant in the context of an automated approach that is already able to deal with the more complicated situation of QCD-induced subtractions, as is the case for MADGrAPH5_AMC@NLO. It is indeed weak corrections that introduce several elements of novelty in our automated approach (see e.g. section 4.3 of ref. [44]); the possibility of testing them in $t \bar{t} H$ production is yet another motivation to pursue the computation we are presenting in this paper.

We point out that, in all cases where both QCD and EW effects are relevant, the structure of the cross section at any given perturbative order (LO, NLO, and so forth) is a linear combination of terms, each of which factorises a coupling-constant factor of the type $\alpha_{s}^{n} \alpha^{m}$, with $n+m$ a constant. Owing to the numerical hierarchy $\alpha \ll \alpha_{S}$, it is natural to organise this combination in decreasing powers of $\alpha_{S}$. The leading term has the largest power of $\alpha_{S}$ and the smallest of $\alpha$, and at the NLO it is identified with QCD corrections. The next term has one power less in $\alpha_{S}$, and an extra one in $\alpha$ : it is what is often identified with EW corrections. This is something of a misnomer, because QCD effects contribute to this term as well, and because it renders difficult the classification of the remaining terms (i.e., beyond the second) in the linear combination mentioned before. Although 
slightly annoying, this is not a major problem, being a question of (naming) conventions and, especially, because the computations of terms beyond the second require a massive effort which one assumes not to be justified in view of the coupling hierarchy. However, if such computations can be performed automatically, no effort will be required, and the validity of that assumption can be explicitly checked. At present, we are facing precisely the situation in which the automated calculation of all the $\alpha_{s}^{n} \alpha^{m}$-proportional terms, both at the $\mathrm{LO}$ and the NLO, is becoming feasible. It is therefore useful to reconsider the general structure of a cross section that involve both strong and EW interactions, and to define more precisely what is dealt with in the context of higher-order computations.

The rest of this paper is organised as follows. In section 2 we discuss the implications of having to treat both QCD and EW effects as small perturbations; although the ideas we introduce are general, we concentrate on $t \bar{t} H$ production to be definite, with further details on its calculation with MAdGRAPH5_AMC@NLO given in section 2.1. In section 3 we present our phenomenological results; in particular, we compare EW and QCD effects at the NLO. We conclude and give our outlook in section 4.

\section{Organisation of the calculation}

The calculation we are carrying out is one where we expand simultaneously in the strong $\left(\alpha_{S}\right)$ and weak $(\alpha)$ coupling constants; this scenario has been called mixed-coupling expansion in ref. [44], a paper whose notation, and in particular that of section 2.4, we shall adopt in what follows. Denoting by $\Sigma\left(\alpha_{S}, \alpha\right)$ a generic observable (e.g., a cross section within cuts, or a histogram bin), in $t \bar{t} H$ production we have, at the Born level:

$$
\Sigma_{t \bar{t} H}^{(\text {Born })}\left(\alpha_{S}, \alpha\right)=\alpha_{S}^{2} \alpha \Sigma_{3,0}+\alpha_{S} \alpha^{2} \Sigma_{3,1}+\alpha^{3} \Sigma_{3,2},
$$

which is a direct consequence of the coupling-constant factors associated with the amplitudes relevant to the three classes of contributing partonic processes, which we list in table 1; samples of the corresponding Feynman diagrams are depicted in figures 1 and 2 . From table 1 one also sees that $\Sigma_{3,1}=\Sigma_{3,2}=0$ in the case of the $g g$-initiated process, while $\Sigma_{3,1}=0$ for the $q \bar{q}$-initiated process with $q \neq b$, owing to the colour structure (proportional to the trace of a single Gell-Mann matrix) of this interference term. When $q=b, \Sigma_{3,1} \neq 0$ because of the contribution of diagrams such as the second one of figure 2, which induce a different colour structure. It has to be pointed out that diagrams of that kind would be present when $q \neq b$ as well, if the CKM matrix featured off-diagonal terms in the third generation; in this work, we have assumed this matrix to be diagonal. At the NLO, we have:

$$
\Sigma_{t \bar{t} H}^{(\mathrm{NLO})}\left(\alpha_{S}, \alpha\right)=\alpha_{S}^{3} \alpha \Sigma_{4,0}+\alpha_{S}^{2} \alpha^{2} \Sigma_{4,1}+\alpha_{S} \alpha^{3} \Sigma_{4,2}+\alpha^{4} \Sigma_{4,3}
$$

which follows from eq. (2.1), since in a QCD-EW mixed-coupling expansion the couplingconstant factors at the NLO are obtained from those relevant to the LO by multiplying them by one power of either $\alpha_{S}$ or $\alpha$ (see eq. (2.23) of ref. [44]).

The notation for the generic short-distance coefficient $\Sigma_{k, q}$ has the following motivation. The integer $k$ is the sum of the powers of $\alpha_{S}$ and $\alpha$ at any given perturbative order; in $t \bar{t} H$ production, $k=3$ at the LO (eq. (2.1)) and $k=4$ at the NLO (eq. (2.2)). This 


\begin{tabular}{|c|c|c|}
\hline Process & $\mathcal{O}(\mathcal{A})$ & $\mathcal{O}(\Sigma)$ \\
\hline$g g \rightarrow t \bar{t} H$ & $\alpha_{s}^{1} \alpha^{1 / 2}$ & $\alpha_{s}^{2} \alpha^{1}$ \\
\hline$q \bar{q} \rightarrow t \bar{t} H, \quad q \neq b$ & $\alpha_{s}^{1} \alpha^{1 / 2}, \alpha^{3 / 2}$ & $\alpha_{s}^{2} \alpha^{1}, \alpha^{3}$ \\
\hline$q \bar{q} \rightarrow t \bar{t} H, \quad q=b$ & $\alpha_{s}^{1} \alpha^{1 / 2}, \alpha^{3 / 2}$ & $\alpha_{s}^{2} \alpha^{1}, \alpha_{s}^{1} \alpha^{2}, \alpha^{3}$ \\
\hline
\end{tabular}

Table 1. Born-level partonic processes relevant to $t \bar{t} H$ production. For each of them, we report the coupling-constant factors in front of the non-null contributions, both at the amplitude (middle column) and at the amplitude squared (rightmost column) level.
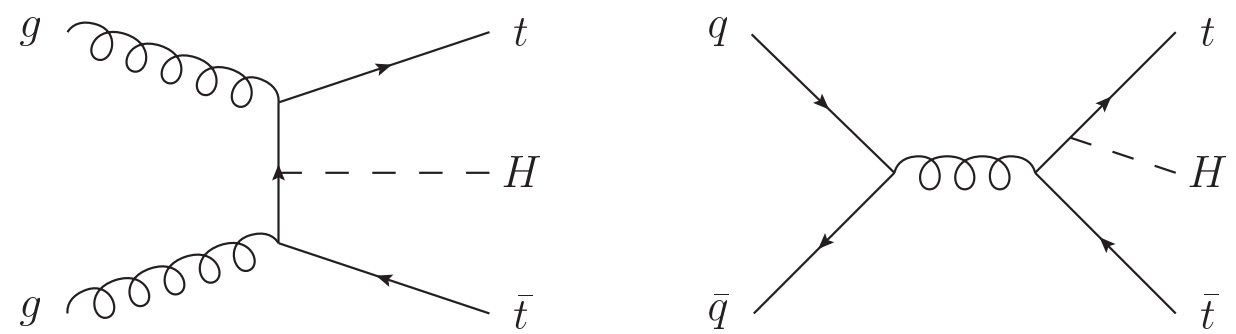

Figure 1. Representative $\mathcal{O}\left(\alpha_{s}^{1} \alpha^{1 / 2}\right)$ Born-level diagrams.
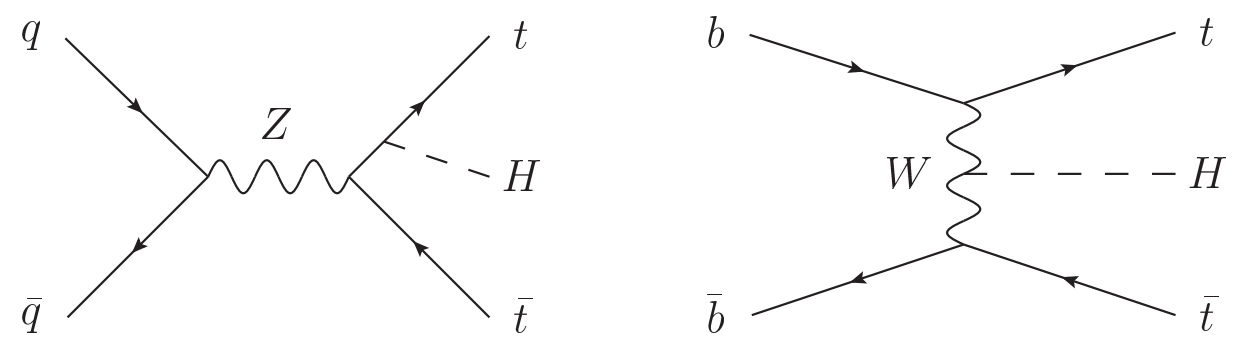

Figure 2. Representative $\mathcal{O}\left(\alpha^{3 / 2}\right)$ Born-level diagrams.

immediately shows that it is also convenient to write $\Sigma_{k, q} \equiv \Sigma_{k_{0}+p, q}$, with $p \geq 0$, for the $\mathrm{N}^{p} \mathrm{LO}$ coefficients; $k_{0}$ is then a fixed, process-specific integer associated with the Born cross section, equal to 3 in $t \bar{t} H$ production. The integer $q$ identifies the various terms of eqs. (2.1) and (2.2). We have conventionally chosen to associate increasing values of $q$ with $\Sigma_{k_{0}+p, q}$ coefficients (at fixed $p$ ) which are increasingly suppressed in terms of the hierarchy of the coupling constants, $\alpha \ll \alpha_{S}$. Thus, $q=0$ corresponds to the coefficient with the largest (smallest) power of $\alpha_{S}(\alpha)$, and conversely for $q=q_{\max }$. This maximum value $q_{\text {max }}$ that can be assumed by $q$ is process- and perturbative-order-dependent, and it grows with the number of amplitudes that interfere and that factorise different coupling-constant combinations; in the case of $t \bar{t} H$ production at the LO, this can be seen by comparing the two rightmost columns of table 1 .

Definition: we propose that the coefficient $\Sigma_{k_{0}+p, q}$ be called the leading (when $q=0$ ), or the $(q+1)^{t h}$-leading (when $q \geq 1$, i.e. second-leading, third-leading, and so forth), term of the $\mathrm{N}^{p} \mathrm{LO}$ contribution to the cross section. ${ }^{1}$ The word "term" may be replaced

\footnotetext{
${ }^{1}$ This classification is the same as that one obtains by counting the powers of $\lambda$ after rescaling $\alpha_{S} \rightarrow \lambda \alpha_{S}$, $\alpha \rightarrow \lambda^{2} \alpha$. Both can be generalised to the case of a mixed-coupling expansion in more than two couplings.
} 


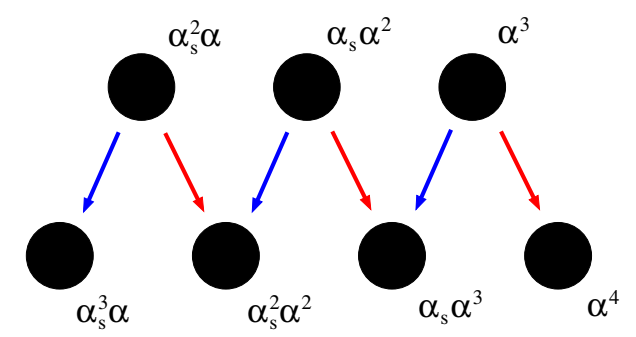

Figure 3. QCD (blue, right-to-left arrows) corrections and EW (red, left-to-right arrows) corrections to $t \bar{t} H$ hadroproduction. See the text for details.

by any suitable synonymous, and in particular by "correction" at the NLO and beyond. We explicitly emphasise that the above convention implies that expressions such as "QCD corrections" or "EW corrections" should be avoided to identify the coefficients $\Sigma_{k_{0}+p, q}$. The key point is that while $\Sigma_{k_{0}+p, q}$ is a well-defined quantity in perturbation theory, QCD corrections or EW corrections are ambiguous concepts (except in two cases, as we shall explain below), which might lead to some confusion.

In order to further the points above, which are valid independently of the process considered, let us restrict to the case of $t \bar{t} H$ production for definiteness. The goal of this paper is that of computing the so-far unknown second-leading NLO correction $\Sigma_{4,1}$ (with some restrictions, to be discussed in section 2.1), and to use it, together with the leading LO and NLO terms, $\Sigma_{3,0}$ and $\Sigma_{4,0}$ respectively, for a sample phenomenology study. The coefficient $\Sigma_{4,0}$ has been available in the literature for a while [17-22, 49], and is traditionally referred to as NLO $Q C D$ corrections; the analogue of the coefficient $\Sigma_{4,1}$, available in the literature for other processes such as $t \bar{t}$ production [35-39] is often referred to as NLO EW corrections. These naming conventions, in their explicit use of "QCD" and "EW", is what we suggest to avoid in the context of a mixed-coupling expansion, and the reason is particularly clear in the case when $\Sigma_{4,1}$ is identified with EW corrections. When doing so, in fact, one implicitly assumes that these are EW corrections just to the leading Born term; furthermore, such corrections cannot be disentangled unambiguously from QCD corrections to the second-leading Born term.

The situation is depicted schematically in figure 3 (which is adapted from ref. [44]): each blob in the upper or lower row corresponds to one of the $\Sigma_{3, q}$ or $\Sigma_{4, q}$ coefficients of eq. (2.1) or eq. (2.2), respectively. We propose that they keywords "QCD corrections" and "EW corrections" be used only to identify the computations that lead to an NLO contribution given a Born contribution, according to the scheme:

$$
\begin{array}{lll}
\alpha_{S}^{n} \alpha^{m} & \stackrel{\mathrm{QCD}}{\longrightarrow} \alpha_{S}^{n+1} \alpha^{m}, \\
\alpha_{S}^{n} \alpha^{m} & \stackrel{\mathrm{EW}}{\longrightarrow} \alpha_{S}^{n} \alpha^{m+1} .
\end{array}
$$

These definitions correspond to the arrows that appear in figure 3: from right to left for QCD corrections, and from left to right for EW corrections. We point out that this terminology is consistent with that typically used in the literature. It only becomes misleading when it is also applied to the coefficients $\Sigma_{k_{0}+1, q}$, because this is equivalent to giving the 

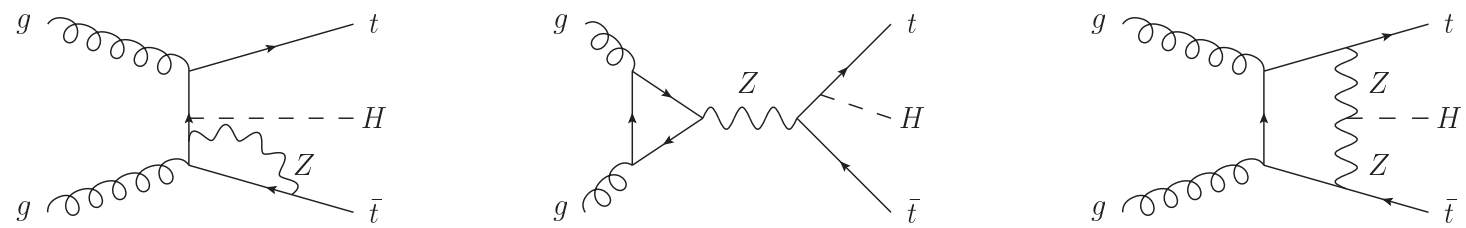

Figure 4. Representative $\mathcal{O}\left(\alpha_{s}^{1} \alpha^{3 / 2}\right)$ one-loop diagrams for the $g g$ channel.

same name to two different classes of objects in figure 3: the blobs and the arrows. If the roles of these two classes are kept distinct, no ambiguity is possible. Consider, for example, the coefficient $\Sigma_{4,1}$ in which we are interested here: it is the second-leading NLO term, which receives contributions both from the EW corrections to the leading Born term $\Sigma_{3,0}$, and from the QCD corrections to the second-leading Born term $\Sigma_{3,1}$.

We note that the discussion given above explains why there is no ambiguity when one works in a single-coupling perturbative expansion. In the case of QCD, for example, the only relevant quantities of figure 3 are the two leftmost blobs (one for each row), and the leftmost arrow. There is thus a one-to-one correspondence between the arrow and the leftmost blob in the lower row: therefore, no confusion arises even if one calls the latter (the leading NLO correction) with the name of the former (the QCD corrections), which is what is usually done. The case of the single-coupling EW expansion is totally analogous, and applies to the quantities that in figure 3 are to the extreme right (namely, $\Sigma_{3,2}, \Sigma_{4,3}$, and the rightmost left-to-right arrow. Note that $\Sigma_{4,1}$ is not involved).

We would like now to elaborate further on the keywords "QCD corrections" and "EW corrections", stressing again the fact that they do not have any deep physical meaning, but may be useful in that they are intuitive, and can be given an operational sense. The best way to do so is that of a constructive bottom-up approach that starts at the level of amplitudes (we note that eqs. (2.3) and (2.4) are at the level of amplitude squared) in order to figure out which contributions each of the coefficients $\Sigma_{4, q}$ receives. While doing so, one needs to bear in mind that, at the NLO, there are two classes of such contributions: those due to real-emission amplitudes (eventually squared), and those due to one-loop amplitudes (eventually contracted with Born amplitudes). Since here we are solely interested in figuring out the general characteristics of the contributions to any given $\Sigma_{4, q}$ (as opposed to performing a complete and explicit computation, which is rather done automatically), the easiest procedure is that of taking representative Born-level diagrams, such as those of figures 1 and 2, and turn them either into one-loop graphs through the insertion of a virtual particle, or into real-emission graphs by emitting one further final-state particle. It is clear that in general it is not possible to obtain all one-loop and real-emission Feynman diagrams in this way (see e.g. the second and third graphs in figure 4), but this is irrelevant for the sake of the present exercise. What is of crucial importance is that, in the context of a mixed QCD-EW expansion, the virtual or final-state particle mentioned before must be chosen in a set larger than the one relevant to a single-coupling series. In particular, for the case of $t \bar{t} H$ production with stable top quarks and Higgs, such a set is:

$$
\left\{g, q, t, Z, W^{ \pm}, H, \gamma\right\}
$$




\begin{tabular}{|cc|cc|}
\hline & Born & $B_{0}=\mathcal{O}\left(\alpha_{s}^{1} \alpha^{1 / 2}\right)$ & $B_{1}=\mathcal{O}\left(\alpha^{3 / 2}\right)$ \\
\hline \multirow{2}{*}{ QCD } & Virtual & $V_{\mathrm{QCD}, 0}=\mathcal{O}\left(\alpha_{s}^{2} \alpha^{1 / 2}\right)$ & $V_{\mathrm{QCD}, 1}=\mathcal{O}\left(\alpha_{s}^{1} \alpha^{3 / 2}\right)$ \\
& Real & $R_{\mathrm{QCD}, 0}=\mathcal{O}\left(\alpha_{s}^{3 / 2} \alpha^{1 / 2}\right)$ & $R_{\mathrm{QCD}, 1}=\mathcal{O}\left(\alpha_{s}^{1 / 2} \alpha^{3 / 2}\right)$ \\
\hline \multirow{2}{*}{ EW } & Virtual & $V_{\mathrm{EW}, 0}=\mathcal{O}\left(\alpha_{s}^{1} \alpha^{3 / 2}\right)$ & $V_{\mathrm{EW}, 1}=\mathcal{O}\left(\alpha^{5 / 2}\right)$ \\
& Real & $R_{\mathrm{EW}, 0}=\mathcal{O}\left(\alpha_{s}^{1} \alpha^{1}\right)$ & $R_{\mathrm{EW}, 1}=\mathcal{O}\left(\alpha^{2}\right)$ \\
\hline
\end{tabular}

Table 2. Coupling-constant factors relevant to Born, one-loop, and real-emission amplitudes; see the text for more details.

where the light quark $q$ may also be a $b$ quark, and the top quark enters only one-loop contributions. In the case of such contributions, the particles in the set of eq. (2.5) are fully analogous to the L-cut particles (see section 3.2.1 of ref. [50]), and we understand ghosts and Goldstone bosons. When the extra particle added to the Born diagram (be it virtual or real) is strongly interacting, it is then natural to classify the resulting one-loop or real-emission diagram as a QCD-type contribution, and a EW-type contribution otherwise. $^{2}$ The idea of this amplitude-level classification is that QCD-type and EW-type contributions will generally lead to QCD and EW corrections at the amplitude-squared level, respectively. However, this correspondence, in spite of being intuitively appealing, is not exact, as we shall show in the following; this is one of the reasons why "QCD corrections" and "EW corrections" must not be interpreted literally. The classification just introduced is used in table 2: for a given Born-level amplitude $B_{i}$ associated with a definite couplingconstant factor, the corresponding one-loop and real-emission quantities are denoted by $V_{\mathrm{QCD}, i}$ and $R_{\mathrm{QCD}, i}$ in the case of QCD-type contributions, and by $V_{\mathrm{EW}, i}$ and $R_{\mathrm{EW}, i}$ in the case of EW-type contributions. We can finally consider all possible combinations $B_{i} \cdot V_{*, j}$, $R_{\mathrm{QCD}, i} \cdot R_{\mathrm{QCD}, j}$, and $R_{\mathrm{EW}, i} \cdot R_{\mathrm{EW}, j}$ and associate them with the relevant amplitude-squared quantities $\Sigma_{4, q}$. Note that one must not consider the $R_{\mathrm{QCD}, i} \cdot R_{\mathrm{EW}, j}$ combinations, owing to the fact that the two amplitudes here are relevant to different final states. ${ }^{3}$

We now observe that this bottom-up construction leads to redundant results. Here, the case in point is that of $V_{\mathrm{QCD}, 1}$ and $V_{\mathrm{EW}, 0}$ : the one-loop diagram (which enters $V_{\mathrm{QCD}, 1}$ ) obtained by exchanging a gluon between the $\bar{q}$ and $\bar{t}$ legs of the diagram to the left of figure 2 is the same diagram as that (which enters $V_{\mathrm{EW}, 0}$ ) obtained by exchanging a $Z$ between the $q$ and intermediate- $t$ legs of the diagram to the right of figure 1 . This fact does not pose any problem in the context of the classification exercise we are carrying out here. Conversely, it is instructive because it shows directly that such contributions cannot be unambiguously given a "QCD correction" or an "EW correction" tag at the level of the cross section, where they will always appear together. We note that a fully similar situation would be that of $R_{\mathrm{QCD}, i} \cdot R_{\mathrm{EW}, j}$, if such quantities were relevant to the present computation; as mentioned in

\footnotetext{
${ }^{2}$ An alternative classification (equivalent to that used here when restricted to $t \bar{t} H$ production and to processes of similar characteristics, but otherwise more general) is one that determines the type of contribution according to the nature of the vertex involved.

${ }^{3}$ For generic processes, this is not necessarily the case, the typical situation being that where some massless particles in the set of eq. (2.5) are present at the Born level.
} 
footnote 3, their analogues will in general contribute to the cross sections of other processes. The final results of our classification exercise are given in eqs. (2.6)-(2.9):

$$
\begin{aligned}
& \Sigma_{4,0} \longleftrightarrow B_{0} \cdot V_{\mathrm{QCD}, 0}, R_{\mathrm{QCD}, 0} \cdot R_{\mathrm{QCD}, 0} \\
& \Sigma_{4,1} \longleftrightarrow B_{0} \cdot\left(V_{\mathrm{QCD}, 1} \oplus V_{\mathrm{EW}, 0}\right), B_{1} \cdot V_{\mathrm{QCD}, 0}, R_{\mathrm{QCD}, 0} \cdot R_{\mathrm{QCD}, 1}, R_{\mathrm{EW}, 0} \cdot R_{\mathrm{EW}, 0}, \\
& \Sigma_{4,2} \longleftrightarrow B_{0} \cdot V_{\mathrm{EW}, 1}, B_{1} \cdot\left(V_{\mathrm{QCD}, 1} \oplus V_{\mathrm{EW}, 0}\right), R_{\mathrm{QCD}, 1} \cdot R_{\mathrm{QCD}, 1}, R_{\mathrm{EW}, 0} \cdot R_{\mathrm{EW}, 1} \\
& \Sigma_{4,3} \longleftrightarrow B_{1} \cdot V_{\mathrm{EW}, 1}, R_{\mathrm{EW}, 1} \cdot R_{\mathrm{EW}, 1} .
\end{aligned}
$$

These equations help summarise the points made above in an explicit manner. For example, we may classify as QCD corrections the second and third terms on the r.h.s. of eq. (2.7), and as EW corrections the fourth term there; as discussed above, the first term is neither of the two. Equations (2.6) and (2.9) receive only QCD and EW corrections, respectively, and indeed they correspond to the results of a single-coupling expansion (in $\alpha_{S}$ and $\alpha$, respectively). The case of eq. (2.8) is fully analogous to that of eq. (2.7). Note finally that, in the equations above and in table 2 , all of the contributions with index " 1 ' vanish identically for the $g g$-initiated process.

The classification procedure carried out above can be extended to any process. This is useful not so much in order to determine which corrections are QCD and which are EW, given the irrelevance of this from the physics viewpoint, but to understand in a quick manner which contributions each of the $\Sigma_{k_{0}+1, q}$ coefficient receives.

\subsection{Calculation of the $\mathcal{O}\left(\alpha_{s}^{2} \alpha^{2}\right)$ contribution with MADGRAPH5_AMC@NLO}

As was stated in section 1 , we are interested in computing $\Sigma_{4,1}$, with the sole exclusion of contributions of QED origin, and thus including in particular weak-only effects. For a generic process or coefficient $\Sigma_{k_{0}+1, q}$, a gauge-invariant separation of NLO EW effects into a QED and a weak subset is not always possible. However, in our case LO diagrams subject to EW corrections do not feature any $W$ boson (note that this is not true for $\Sigma_{4,2}$ and $\Sigma_{4,3}$ ); furthermore, no triple gauge-vector vertex appears in one-loop diagrams. Thus, weak-only NLO corrections to $t \bar{t} H$ hadroproduction are well defined.

The first implication of restricting oneself to weak-only contributions is that of removing the photon from the set of eq. (2.5). At the level of real-emission diagrams, this implies that no graphs with external photons will contribute to our results; this also simplifies the structure of the subtractions, which is identical to that of a pure-QCD computation, in view of the absence of soft and collinear singularities of QED origin. The removal of the photon contributions from real-emission matrix elements must have a consistent counterpart at the level of one-loop amplitudes. In order to discuss this matter, we remind the reader that all computations performed by MADGRAPH5_AMC@NLO are based on a UFO [51] model, that encodes the basic information on the Lagrangian of the relevant theory. For NLO computations, in particular, on top of the usual Feynman rules one also needs those for the $R_{2}$ counterterms [52] and for the UV counterterms. Two UFO models are available that allow one to perform QCD+EW corrections in the SM; our default (used for the majority of the results to be presented in section 3$)$ is that which adopts the $\alpha\left(m_{Z}\right)$ renormalisation scheme [53] (and thus $\alpha\left(m_{Z}\right), m_{Z}$, and $m_{W}$ as input parameters), while an alternative one 


\begin{tabular}{|c|c|}
\hline \multicolumn{2}{|c|}{ Virtual corrections } \\
\hline$B_{0} \cdot\left(V_{\mathrm{QCD}, 1} \oplus V_{\mathrm{EW}, 0}\right)$ & $g g \rightarrow t \bar{t} H$ \\
& $q \bar{q} \rightarrow t \bar{t} H$ \\
\hline$B_{1} \cdot V_{\mathrm{QCD}, 0}$ & $q \bar{q} \rightarrow t \bar{t} H$ \\
\hline \multicolumn{2}{|c|}{ Real-emission corrections } \\
\hline$R_{\mathrm{QCD}, 0} \cdot R_{\mathrm{QCD}, 1}$ & $q \bar{q} \rightarrow t \bar{t} H g$ \\
& $q g \rightarrow t \bar{t} H q$ \\
\hline & $g g \rightarrow t \bar{t} H Z$ \\
& $q \bar{q} \rightarrow t \bar{t} H Z$ \\
$R_{\mathrm{EW}, 0} \cdot R_{\mathrm{EW}, 0}$ & $q \bar{q}^{\prime} \rightarrow t \bar{t} H W$ \\
& $g g \rightarrow t \bar{t} H H$ \\
& $q \bar{q} \rightarrow t \bar{t} H H$ \\
\hline
\end{tabular}

Table 3. List of partonic processes that contribute to the second-leading NLO term $\Sigma_{4,1}$, according to the classification given in table 2 and eq. (2.7). See the text for more details.

implements the $G_{\mu}$ scheme $[53,54]$ (where the input parameters are $G_{F}$, measured in $\mu$ decays, $m_{Z}$, and $m_{W}$ ); masses and wave functions are renormalised on-shell. For both models, the $R_{2}$ rules have been taken from refs. [55-57]. In view of the complexity of the models, all counterterms have been cross-checked with an independent Matнeмatica package. Having the full QCD+EW corrections available in the models, one can rather easily exclude the photon contributions to loop diagrams at generation time, as well as from masses and wave-function UV counterterms, and from $R_{2}$ counterterms, thanks to the extreme flexibility of MADLOOP5 (see section 2.4.2 of ref. [44], and in particular the concept of loop-content filtering there, for more informations). The result of this procedure has been validated by computing with MADGRAPH5_AMC@NLO the complete weak-only contributions to $p p \rightarrow t \bar{t}$ production, and by comparing it (at the level of differential distributions) to what we have obtained for this process with Feynarts [58], Formcalc [59], and LoopTools [60]. Furthermore, these tools have also been used for computing the virtual weak contributions to $H H \rightarrow t \bar{t}$ production, as a way to cross-check the renormalisation of the $t \bar{t} H$ Yukawa and its use in MADGraPh5_AMC@NLO; again, an excellent agreement has been found.

In table 3 we list explicitly all the partonic processes that contribute to the $\mathcal{O}\left(\alpha_{s}^{2} \alpha^{2}\right)$ coefficient $\Sigma_{4,1}$. Each process understands the computation of the corresponding amplitudes in the left column of the table, according to the classification given in table 2. So for example for the first real-emission process of table 3 , the contribution to $\Sigma_{4,1}$ is given by the $\mathcal{O}\left(\alpha_{s}^{3 / 2} \alpha^{1 / 2}\right) q \bar{q} \rightarrow t \bar{t} H g$ tree-level amplitude times the $\mathcal{O}\left(\alpha_{s}^{1 / 2} \alpha^{3 / 2}\right) q \bar{q} \rightarrow t \bar{t} H g$ tree-level amplitude.

Loop diagrams of $\mathcal{O}\left(\alpha_{s}^{2} \alpha^{1 / 2}\right)\left(V_{\mathrm{QCD}, 0}\right)$ enter both the first- and the second-leading NLO terms, $\Sigma_{4,0}$ and $\Sigma_{4,1}$. However, in the latter case the interference with the $\mathcal{O}\left(\alpha^{3 / 2}\right)$ Born amplitude $B_{1}$ (see eq. (2.7)) is such that self-energy and vertex corrections vanish owing to 
the colour structure; thus, only boxes and pentagons contribute, and UV divergencies of QCD origin are not present at $\mathcal{O}\left(\alpha_{s}^{2} \alpha^{2}\right)$. Furthermore, the $g g$-initiated virtual corrections are also soft- and collinear-finite; consistently, as one can see from table 3 , there is no realemission counterpart which might cancel such divergencies. This is not the case for the $q \bar{q}$ process, where cancellations of singularities do occur between the virtual and real-emission processes. When $q \neq b$, such singularities are only of soft origin, owing again to the colour structure of the diagrams involved, which implies that emissions of the gluon from an initial-state leg both on the left and on the right of the Cutkosky cut give a vanishing contribution. This is consistent with the fact that for such light quark the $\mathcal{O}\left(\alpha_{s}^{1} \alpha^{2}\right)$ Bornlevel cross section is zero (see table 1 ), since this cross section would have to factorise (times the relevant Altarelli-Parisi kernel [61]) in the case of collinear singularities. Similar considerations (and the absence of virtual contributions) lead to the conclusion that the $q g$-initiated real-emission process is also soft- and collinear-finite. Finally, the real-emission contributions of weak origin $\left(R_{\mathrm{EW}, 0} \cdot R_{\mathrm{EW}, 0}\right)$ are finite everywhere in the phase space.

We conclude this section by outlining the ingredients that enter the results that we shall present in section 3 , and which are mainly based on the coefficients $\Sigma_{3,0}$ (at the LO), $\Sigma_{4,0}$, and $\Sigma_{4,1}$ (at the NLO). The calculation of the former two coefficients is the same as that which has already appeared in refs. [21, 44], and is fully automated in MADGraPh5_AMC@NLO. We remind the reader that MADGRAPH5_AMC@NLO contains all ingredients relevant to the computations of LO and NLO cross sections, with or without matching to parton showers. NLO results not matched to parton showers are obtained by adopting the FKS method $[62,63]$ for the subtraction of the singularities of the realemission matrix elements (automated in the module MADFKS [64]), and the OPP integralreduction procedure [65] for the computation of the one-loop matrix elements (automated in the module MADLOop [50], which makes use of CuTTools [66] and of an in-house implementation of the representation proposed in ref. [67] (OpenLoops)). The automation of the mixed-coupling expansions has not been completely validated ${ }^{4}$ yet in MADFKS, but is fully operational in MADLOOP5. Thus, the calculation of $\Sigma_{4,1}$ has been achieved by constructing "by hand", for the specific process we are considering, the IR counterterms relevant to the subtraction of QCD singularities; this operation will serve as a benchmark when the automation of the subtractions in a mixed-coupling scenario will be achieved. Apart from this, all of the other relevant procedures, and in particular the generation of the matrix elements and of the $\mathcal{S}$ functions (which achieve the dynamic phase-space partition needed in FKS), are automated.

Given that the subtraction of the IR singularities that affect $\Sigma_{4,1}$ is not completely automated, we have simplified the calculation by ignoring the contribution to this coefficient due to $b \bar{b}$-initiated partonic processes (as was discussed before, this process is the only one where initial-state collinear singularities appear, and thus no collinear subtractions are needed in our computation). This approximation is fully justified numerically, in view of

\footnotetext{
${ }^{4}$ This validation consists only in addressing bookkeeping issues, given that QED subtractions are a simpler version of their QCD counterparts, as was already pointed out in section 1, and that QCD subtractions relevant to the beyond-leading $\Sigma_{k_{0}+1, q}$ coefficients $(q \geq 1)$ are fully analogous to those, already automated, relevant to the leading term $\Sigma_{k_{0}+1,0}$.
} 


\begin{tabular}{|c|c|c|}
\hline Label & Meaning & Restrictions \\
\hline LO or Born & $\alpha_{S}^{2} \alpha \Sigma_{3,0}$ & \\
NLO QCD & $\alpha_{S}^{3} \alpha \Sigma_{4,0}$ & \\
NLO weak & $\alpha_{S}^{2} \alpha^{2} \Sigma_{4,1}$ & no QED, no $b \bar{b} \rightarrow t \bar{t} H+X$, no $p p \rightarrow t \bar{t} H+V$ \\
HBR & $\alpha_{S}^{2} \alpha^{2} \Sigma_{4,1}$ & no QED, no $b \bar{b} \rightarrow t \bar{t} H+X$, only $p p \rightarrow t \bar{t} H+V$ \\
\hline
\end{tabular}

Table 4. Shorthand notation that we shall use in section 3. HBR is an acronym for Heavy Boson Radiation. $V$ stands for a Higgs, a $W$, or a $Z$, and HBR understands the sum of the corresponding three cross sections. The reader is encouraged to check section 2 for the precise definitions of all the quantities involved.

the extremely small $b \bar{b} \rightarrow t \bar{t} H$ cross section at the LO, which we shall report in section 3 . We shall also present the contributions of the $R_{\mathrm{EW}, 0} \cdot R_{\mathrm{EW}, 0}$ processes (see table 3 ) separately from the rest, in keeping with what is usually done in the context of EW computations. We emphasise that, as the general derivation presented before shows, there is no real motivation for ignoring such contributions completely. The argument that an extra final-state boson can be tagged might be made, but only in the context of a fully realistic analysis (since bosons cannot be seen directly in a detector), which is beyond the scope of the present paper. We note that the corresponding cross section is not negligible, as we shall document in section 3; our results, being inclusive in the extra boson, represent an upper bound for those obtained by applying proper acceptance cuts.

In table 4 we give the shorthand naming conventions that we shall adopt in section 3 . We use names which are similar to those most often used in the context of EW higherorder computations, so as to facilitate the reading of the phenomenological results. As was discussed at length in the present section, the contents of the various terms are more involved than their names may suggest, and we refer the reader to such a section for the necessary definitions.

\section{Results}

In this section we present a sample of results obtained by simulating $t \bar{t} H$ production in $p p$ collisions at three different collider c.m. energies: 8, 13, and $100 \mathrm{TeV}$. We have chosen the top-quark and Higgs masses as follows:

$$
m_{t}=173.3 \mathrm{GeV}, \quad m_{H}=125 \mathrm{GeV},
$$

and adopted the MSTWnlo2008 [68] PDFs with the associated $\alpha_{S}\left(m_{Z}\right)$ for all NLO as well as LO predictions (since we are chiefly interested in assessing effects of matrix-element origin). In our default $\alpha\left(m_{Z}\right)$-scheme, the EW coupling constant is [69]:

$$
\frac{1}{\alpha\left(m_{Z}\right)}=128.93
$$


The central values of the renormalisation $\left(\mu_{R}\right)$ and factorisation $\left(\mu_{F}\right)$ scales have been taken equal to the reference scale:

$$
\mu=\frac{H_{T}}{2} \equiv \frac{1}{2} \sum_{i} \sqrt{m_{i}^{2}+p_{T}^{2}(i)}
$$

where the sum runs over all final-state particles. The theoretical uncertainties due to the $\mu_{R}$ and $\mu_{F}$ dependencies that affect the coefficient $\Sigma_{4,0}$ have been evaluated by varying these scales independently in the range:

$$
\frac{1}{2} \mu \leq \mu_{R}, \mu_{F} \leq 2 \mu,
$$

and by keeping the value of $\alpha$ fixed. The calculation of this theory systematics does not entail any independent runs, being performed through the reweighting technique introduced in ref. [70], which is fully automated in MADGRAPH5_AMC@NLO. All the input parameters not explicitly mentioned here have been set equal to their PDG values [71].

We shall consider two scenarios: one where no final-state cuts are applied (i.e. fully inclusive), and a "boosted" one, generally helpful to reduce the contamination of light-Higgs signals due to background processes [72-74], where the following cuts

$$
p_{T}(t) \geq 200 \mathrm{GeV}, \quad p_{T}(\bar{t}) \geq 200 \mathrm{GeV}, \quad p_{T}(H) \geq 200 \mathrm{GeV},
$$

are imposed; since these emphasise the role of the high- $p_{T}$ regions, the idea is that of checking whether weak effects will have a bigger impact there than in the whole of the phase space. We shall report in section 3.1 our predictions for total rates, for the three collider c.m. energies and in both the fully inclusive and the boosted scenario. In section 3.2 several differential distributions will be shown, at a c.m. of $13 \mathrm{TeV}$ with and without the cuts of eq. (3.5), and at a c.m. of $100 \mathrm{TeV}$ in the fully-inclusive case only.

Throughout this section, we shall make use of the shorthand notation introduced at the end of section 2 - see in particular table 4.

\subsection{Inclusive rates}

In this section we present our predictions for inclusive rates, possibly within the cuts of eq. (3.5). As was already stressed, the results for the LO and NLO QCD contributions are computed in the same way as has been done previously with MADGraPH5_AMC@NLO or its predecessor AMC@NLO in refs. [21, 44]. There are small numerical differences $(\mathcal{O}(3 \%))$ with ref. [44], which are almost entirely due to the choice of the value of $\alpha$, and to a very minor extent to that of $m_{t}$. As far as ref. [21] is concerned, different choices had been made there for the top and Higgs masses, and for the reference scale.

The predicted rates (in $\mathrm{pb}$ ) are given in table 5; the values outside parentheses are the fully-inclusive ones, while those in parentheses are relevant to the boosted scenario; in both cases, the NLO QCD contributions are sizable and positive. As far as the NLO weak contributions are concerned, they are negative and in absolute value rather small in the fully inclusive case, although their relative impact w.r.t. that of QCD tends to increase with the collider energy. This picture is reversed (i.e. the impact slightly decreases) in the 


\begin{tabular}{|l|ccc|}
\hline$\sigma(\mathrm{pb})$ & \multicolumn{1}{|c}{$8 \mathrm{TeV}$} & $13 \mathrm{TeV}$ & $100 \mathrm{TeV}$ \\
\hline $\mathrm{LO}$ & $1.001 \cdot 10^{-1}\left(2.444 \cdot 10^{-3}\right)$ & $3.668 \cdot 10^{-1}\left(1.385 \cdot 10^{-2}\right)$ & $24.01(2.307)$ \\
\hline NLO QCD & $2.56 \cdot 10^{-2}\left(4.80 \cdot 10^{-4}\right)$ & $1.076 \cdot 10^{-1}\left(3.31 \cdot 10^{-3}\right)$ & $9.69(0.902)$ \\
\hline NLO weak & $-1.22 \cdot 10^{-3}\left(-2.04 \cdot 10^{-4}\right)$ & $-6.54 \cdot 10^{-3}\left(-1.14 \cdot 10^{-3}\right)$ & $-0.712(-0.181)$ \\
\hline
\end{tabular}

Table 5. LO, NLO QCD, and NLO weak contributions to the total rate (in pb), for three different collider energies. The results in parentheses are relevant to the boosted scenario, eq. (3.5).

\begin{tabular}{|l|ccc|}
\hline$\delta_{\mathrm{NLO}}(\%)$ & $8 \mathrm{TeV}$ & $13 \mathrm{TeV}$ & $100 \mathrm{TeV}$ \\
\hline $\mathrm{QCD}$ & $+25.6_{-11.8}^{+6.2}\left(+19.6_{-11.0}^{+3.7}\right)$ & $+29.3_{-11.6}^{+7.4}\left(+23.9_{-11.2}^{+5.4}\right)$ & $+40.4_{-11.6}^{+9.9}\left(+39.1_{-10.4}^{+9.7}\right)$ \\
\hline weak & $-1.2(-8.3)$ & $-1.8(-8.2)$ & $-3.0(-7.8)$ \\
\hline
\end{tabular}

Table 6. NLO QCD and weak contributions, as fractions of the corresponding LO cross section. The results in parentheses are relevant to the boosted scenario, eq. (3.5). In the case of QCD, the results of scale variations are also shown.

boosted scenario, ${ }^{5}$ where on the other hand the absolute values of the weak contributions are non-negligible. These features can be understood more directly by looking at the NLO contributions as fractions ${ }^{6}$ of the corresponding LO cross section; they are reported in this form in table 6. In that table, the entries of the first (second) row are the ratios of the entries in the second (third) row over those in the first row of table 5. One sees that the QCD contributions increase the LO cross sections by $25 \%(20 \%)$ to $40 \%$, while the weak ones decrease it by $1 \%$ to $3 \%$ in the fully-inclusive case, and by $8 \%$ when the cuts of eq. (3.5) are applied. In the first row of table 6 we also report (by using the usual "error" notation) the fractional scale uncertainty that affects the LO+NLO QCD rates. This is computed by taking the envelope of the cross sections that result from the scale variations as given in eq. (3.4), and by dividing it by the LO predictions obtained with central scales. Note that this is not the usual way of presenting the scale systematics (which entails using the central $\mathrm{LO}+\mathrm{NLO}$ prediction as a reference), and thus the results of table 6 might seem, at the first glance, to be larger than those reported in ref. [44], but are in fact perfectly consistent with those. Our choice here is motivated by the fact that, by using the LO cross sections as references, we can compare NLO QCD and weak effects on a similar footing. The main message of table 6 is, then, that in the fully inclusive case the weak contributions are entirely negligible in view of the scale uncertainties that affect the numerically-dominant LO+NLO QCD cross sections. On the other hand, in the boosted scenario they become comparable with the latter, and they must thus be taken into account. This feature will also be evident when differential distributions will be considered (see section 3.2).

The impacts of the individual partonic channels on the NLO weak contributions are reported in table 7 , still as fractions of the LO cross sections - hence, the sum of all the

\footnotetext{
${ }^{5}$ Having said that, we also remark that the cuts of eq. (3.5) are imposed irrespective of the collider energy. By increasing the c.m. energy, one would have to increase the required minimal $p_{T}$ 's in order to have similarly boosted configurations.

${ }^{6}$ The statistics we have employed in the computation of the cross sections is such that the typical error affecting such fractions, in the present and forthcoming tables, is of the order of $0.1 \%$.
} 


\begin{tabular}{|l|ccc|}
\hline$\delta_{\mathrm{NLO}}(\%)$ & $8 \mathrm{TeV}$ & $13 \mathrm{TeV}$ & $100 \mathrm{TeV}$ \\
\hline$g g$ & $-0.67(-2.9)$ & $-1.12(-4.0)$ & $-2.64(-6.8)$ \\
\hline$u \bar{u}$ & $-0.01(-3.2)$ & $-0.15(-2.3)$ & $-0.10(-0.5)$ \\
\hline$d \bar{d}$ & $-0.55(-2.2)$ & $-0.52(-1.9)$ & $-0.23(-0.5)$ \\
\hline$u g$ & $+0.03(+0.02)$ & $+0.03(+0.01)$ & $+0.01(<0.01)$ \\
\hline$d g$ & $-0.02(-0.01)$ & $-0.02(-0.01)$ & $-0.01(>-0.01)$ \\
\hline
\end{tabular}

Table 7. Breakdowns per partonic channel of the results of table 6 for the NLO weak contributions. The results in parentheses are relevant to the boosted scenario, eq. (3.5). By $u$ and $d$ we understand $c$ and $s$ as well, respectively. By $u g$ and $d g$ we understand $\bar{u} g$ and $\bar{d} g$ as well, respectively.

entries in a given column of table 7 is equal to the entry in the same column and in the last row of table 6 . We point out that this breakdown into individual partonic contributions, which is rather commonly shown in the context of EW calculations, is unambiguous because QCD-induced singularities are only of soft type (see section 2.1), and thus real-emission matrix elements and their associated Born-like counterparts have the same initial-state partons. From table 7 we see, as is expected, that the dominance of the $g g$ channel, which is moderate at $8 \mathrm{TeV}$, rapidly increases with the collider c.m. energy. This trend is mitigated when the cuts of eq. (3.5) are applied, to the extent that, at the LHC, the $u \bar{u}+d \bar{d}$ cross section is larger than or comparable to the $g g$ one: the boosted scenario forces the Bjorken $x$ 's to assume larger values, where the quark densities are of similar size as that of the gluon.

We now turn to considering the contributions due to processes that feature an extra weak boson in the final state, on top of the Higgs which is present by definition; we remind the reader that these contributions have been denoted by HBR (see table 4). The relevant results are shown in table 8 , as fractions of the corresponding LO cross section; hence, they are directly comparable to the last row of table 6 . Note that, in the case of the $t \bar{t} H H$ final state, a kinematic configuration contributes to the boosted scenario provided that the Higgs- $p_{T}$ cut of eq. (3.5) is satisfied for at least one of the two Higgses. From tables 8 and 6 , one sees that the HBR and NLO weak contributions, in the case of the fully-inclusive cross sections, tend to cancel each other to a good extent: at the $75 \%, 50 \%$, and $30 \%$ level at 8,13 and $100 \mathrm{TeV}$ respectively. This is not true in the boosted scenario: although the HBR cross sections grow faster than the LO ones (being $0.9 \%$ of the latter in the fully-inclusive case, and $1.7 \%$ in the boosted one), their growth is slower than that of their NLO-weak counterparts. Both contributions feature Sudakov logarithms, but we point out that the overall scaling behaviour in hadronic collisions is determined, among other things, by the complicated interplay between that of the matrix elements, and the parton luminosities; the latter are not the same in the case of the NLO-weak and HBR contributions. This has several consequences. For example, we note that the relative individual contributions to the HBR cross sections behave differently with the collider energy: the $W$-emission contribution decreases, while the $Z$ - and $H$-emission ones increase, owing to the presence of $g g$-initiated partonic processes. Furthermore, the growth of PDFs at small $x$ 's implies that processes are closer to threshold than the collider energy would naively imply, and thus the phase-space suppres- 


\begin{tabular}{|l|ccc|}
\hline$\delta_{\text {HBR }}(\%)$ & $8 \mathrm{TeV}$ & $13 \mathrm{TeV}$ & $100 \mathrm{TeV}$ \\
\hline$W$ & $+0.42(+0.74)$ & $+0.37(+0.70)$ & $+0.14(+0.22)$ \\
\hline$Z$ & $+0.29(+0.56)$ & $+0.34(+0.68)$ & $+0.51(+0.95)$ \\
\hline$H$ & $+0.17(+0.43)$ & $+0.19(+0.48)$ & $+0.25(+0.53)$ \\
\hline sum & $+0.88(+1.73)$ & $+0.90(+1.86)$ & $+0.90(+1.70)$ \\
\hline
\end{tabular}

Table 8. Contributions due to $W, Z$, and $H$ radiation, as fractions of the corresponding LO cross section. The results in parentheses are relevant to the boosted scenario, eq. (3.5).

sion due to the presence of an extra massive particle in the HBR processes is not negligible. Finally, this mass effect also implies that the Bjorken $x$ 's relevant to HBR are slightly larger than those relevant to the NLO-weak contributions, and are thus associated on average with slightly smaller luminosity factors. As was already discussed in section 2.1, the results of table 8 are an upper bound for the HBR contributions when these are subject to extra bosontagging conditions, which have not been considered here. On the other hand, nothing prevents one from defining the $t \bar{t} H$ cross section inclusively in any extra weak-boson radiation; given the opposite signs of the NLO-weak and HBR cross sections, this may possibly be beneficial (for example, if constraining or measuring $\lambda_{t \bar{t} H}$ ). Such a definition is fully consistent with perturbation theory, since both HBR and NLO-weak contributions are of $\mathcal{O}\left(\alpha_{s}^{2} \alpha^{2}\right)$.

All the results presented so far have been obtained in the $\alpha\left(m_{Z}\right)$ scheme. It is therefore interesting to check what happens by considering the $G_{\mu}$ scheme, which entails a different renormalisation procedure and different inputs. In such a scheme we have (at the LO):

$$
\frac{1}{\alpha}=132.23 \text {. }
$$

The LO results are presented in the first row of table 9 ; the second row displays the relative difference w.r.t. their $\alpha\left(m_{Z}\right)$-counterparts of table 5 :

$$
\Delta_{\mathrm{LO}}^{G_{\mu}}=\frac{\mathrm{LO}-\mathrm{LO}^{G_{\mu}}}{\mathrm{LO}} .
$$

The latter figures constitute a simple cross check: given that the LO cross section factorises $\alpha_{S}^{2} \alpha$, at this perturbative order the difference can only be due to the values of the EW coupling constant, and the $2.5 \%$ reported in table 9 is the difference ${ }^{7}$ between the $\alpha$ 's of eqs. (3.6) and (3.2). Therefore, at the LO the EW-scheme dependence of the cross section is larger than (at the LHC) or comparable to (at $100 \mathrm{TeV}$ ) the NLO weak contribution in the fully-inclusive case, while it is about a third of the latter in the boosted scenario. When NLO corrections are included, however, things do change. In the $G_{\mu}$ scheme, the NLO weak contributions are positive for the fully-inclusive rates, at variance with the $\alpha\left(m_{Z}\right)$ scheme; see the third row of table 9 , where they are reported as fractions of the LO cross sections:

$$
\delta_{\text {weak }}^{G_{\mu}}=\frac{\mathrm{NLO}_{\text {weak }}^{G_{\mu}}}{\mathrm{LO}^{G_{\mu}}}
$$

\footnotetext{
${ }^{7}$ The $\alpha\left(m_{Z}\right)$ - and $G_{\mu}$-scheme runs have been performed with different statistics and seeds, so that other small differences are present.
} 


\begin{tabular}{|r|ccc|}
\hline & $8 \mathrm{TeV}$ & $13 \mathrm{TeV}$ & $100 \mathrm{TeV}$ \\
\hline $\mathrm{LO}^{G_{\mu}}(\mathrm{pb})$ & $9.758 \cdot 10^{-2}\left(2.382 \cdot 10^{-3}\right)$ & $3.575 \cdot 10^{-1}\left(1.351 \cdot 10^{-2}\right)$ & $23.41(2.249)$ \\
\hline$\Delta_{\mathrm{LO}}^{G_{\mu}}(\%)$ & $+2.5(+2.5)$ & $+2.5(+2.5)$ & $+2.5(+2.5)$ \\
\hline$\delta_{\text {weak }}^{G_{\mu}}(\%)$ & $+1.8(-5.1)$ & $+1.3(-4.9)$ & $+0.1(-4.5)$ \\
\hline$\Delta_{\mathrm{LO}+\mathrm{NLO}}^{G_{\mu}}(\%)$ & $-0.5(-0.9)$ & $-0.5(-1.1)$ & $-0.6(-1.0)$ \\
\hline
\end{tabular}

Table 9. Results in $G_{\mu}$-scheme: Born cross sections, relative differences $(\Delta)$ w.r.t. those obtained in the $\alpha\left(m_{Z}\right)$ scheme, and fractional NLO-weak contribution $(\delta)$. The results in parentheses are relevant to the boosted scenario, eq. (3.5).

i.e. they are the analogues of the quantities in the second row of table 6. More importantly, the differences between the two schemes for the NLO-accurate weak cross sections are much reduced w.r.t. those at the $\mathrm{LO}$. This is documented in the fourth row of table 9 , where we show the values of:

$$
\Delta_{\mathrm{LO}+\mathrm{NLO}}^{G_{\mu}}=\frac{\mathrm{LO}+\mathrm{NLO}_{\text {weak }}-\left(\mathrm{LO}^{G_{\mu}}+\mathrm{NLO}_{\text {weak }}^{G_{\mu}}\right)}{\mathrm{LO}+\mathrm{NLO}_{\text {weak }}}
$$

which are smaller in absolute value than their LO counterparts, and whose independence of the collider energy is remarkable. Thus, in the boosted case one sees that the fact that weak contributions have a significant impact on NLO-accurate cross sections is a conclusion that holds true in both of the EW schemes adopted in this paper.

We conclude this section with a brief discussion on the impact of the $b \bar{b}$-initiated contributions, which we have ignored in our NLO-accurate results, for the reasons explained in section 2.1. In table 10 we present the contributions to the Born cross sections (again for the input parameters relevant to the $\alpha\left(m_{Z}\right)$ scheme) due to all the relevant couplingconstant factors (see eq. (2.1)); we remind the reader than only the $\alpha_{S}^{2} \alpha$ term is included in the LO predictions shown so far. The $b \bar{b}$ contribution to $\Sigma_{3,0}$ appears in fact to be quite irrelevant (being at most $0.36 \%$ at $100 \mathrm{TeV}$ ); those to $\Sigma_{3,1}$ and $\Sigma_{3,2}$ are comparable or slightly larger in absolute value, and furthermore they tend to cancel each other. Given that there is no mechanism at the NLO that could enhance the $b \bar{b}$-initiated cross section in a much stronger way than for the other partonic contributions at the same order, our assumption appears to be perfectly safe. It is thus of academic interest the fact that the results for the $b \bar{b}$-induced $\Sigma_{3, q}$ coefficients do not obey the numerical hierarchy suggested by their corresponding coupling-constant factors (which hierarchy is violated owing to the opening of $t$-channel diagrams, such as the one on the right of figure 2). When the mixed-coupling expansion will be fully automated in MADGRAPH5_AMC@NLO, one will easily verify whether such a feature survives NLO corrections. We finally remark that the contributions to $\alpha^{3} \Sigma_{3,2}$ not due to a $b \bar{b}$ initial state are very small, and do respect the hierarchy mentioned above: expressed as fractions of $\alpha_{S}^{2} \alpha \Sigma_{3,0}$, they amount to $0.1 \%$, $0.08 \%$, and $0.01 \%$ at 8,13 , and $100 \mathrm{TeV}$ respectively. Together with the results of table 10 , this implies that the $\alpha^{3} \Sigma_{3,2}$ total cross section, summed over all initial states, is a fraction of the $\alpha_{S}^{2} \alpha \Sigma_{3,0}$ one equal to $0.4 \%, 0.5 \%$, and $0.8 \%$ at the three collider energies considered 


\begin{tabular}{|c|ccc|}
\hline$\sigma_{b \bar{b} \rightarrow t \bar{t} H}(\mathrm{pb})$ & $8 \mathrm{TeV}$ & $13 \mathrm{TeV}$ & $100 \mathrm{TeV}$ \\
\hline$\alpha_{S}^{2} \alpha \Sigma_{3,0}$ & $1.8 \cdot 10^{-4}$ & $9.1 \cdot 10^{-4}$ & $8.6 \cdot 10^{-2}$ \\
\hline$\alpha_{S} \alpha^{2} \Sigma_{3,1}$ & $-1.3 \cdot 10^{-4}$ & $-1.5 \cdot 10^{-3}$ & $-1.3 \cdot 10^{-1}$ \\
\hline$\alpha^{3} \Sigma_{3,2}$ & $3.1 \cdot 10^{-4}$ & $1.6 \cdot 10^{-3}$ & $1.9 \cdot 10^{-1}$ \\
\hline
\end{tabular}

Table 10. Leading, second-leading, and third-leading Born contributions due to the $b \bar{b}$ initial state.

in this paper. This is at most (at $8 \mathrm{TeV}$ ) a third of the NLO weak contribution (in the $\alpha\left(m_{Z}\right)$ scheme), and is not enhanced in the boosted regime: it is thus quite negligible, which is the reason why we have ignored it.

\subsection{Differential distributions}

We now turn to presenting results for differential distributions. In order to be definite, we have considered the following observables: the transverse momenta of the Higgs $\left(p_{T}(H)\right)$, top quark $\left(p_{T}(t)\right)$, and $t \bar{t}$ pair $\left(p_{T}(t \bar{t})\right)$, the invariant mass of the $t \bar{t} H$ system $(M(t \bar{t} H))$, the rapidity of the top quark $(y(t))$, and the difference in rapidity between the $t \bar{t}$ pair and the Higgs boson $(\Delta y(t \bar{t}, H))$. The corresponding six distributions are shown at a collider energy of $13 \mathrm{TeV}$ (figure 5), $100 \mathrm{TeV}$ (figure 6), and $13 \mathrm{TeV}$ in the boosted scenario of eq. (3.5) (figure 9). In the case of the HBR process $p p \rightarrow t \bar{t} H H$, owing to the inclusive (in the two Higgses) definition of the latter the histograms relevant to the observables that depend explicitly on the Higgs four-momentum (i.e., $p_{T}(H), M(t \bar{t} H)$, and $\left.\Delta y(t \bar{t}, H)\right)$ may receive up to two entries per event.

Figures 5, 6, and 9 have identical layouts. The main frame displays three distributions, which correspond to the LO (black dashed), LO+NLO QCD (red solid, superimposed with full circles), and $\mathrm{LO}+\mathrm{NLO} \mathrm{QCD}+\mathrm{NLO}$ weak (green solid) cross sections. The latter two distributions are therefore the bin-by-bin analogues of the sum of the upper two entries and of the sum of the three entries, respectively, in a given column of table 5. The middle inset presents the ratios of the two NLO-accurate predictions over the corresponding LO one - these are therefore the $K$ factors. Centered around the NLO QCD $K$ factor we show a mouse-grey band, which represents the fractional scale uncertainty, defined in full analogy to what has been done in table 6 . Finally, the lower inset displays the ratios of the NLO QCD, NLO weak, and HBR (dot-dashed magenta) contributions over the LO cross section - these are therefore the analogues of the first two lines of table 6 and of the last line of table 8 , respectively.

Further details on the NLO weak and HBR results relevant to figures 5 and 6 are given in figures 7 and 8, respectively. The main frames display the cross sections, and in the case of the NLO weak contributions the individual results for the three dominant partonic channels (namely, $g g, d \bar{d}$, and $u \bar{u}$ ) are also shown. The lower insets contain the same information, but in the form of fractions over the relevant LO cross sections; these are thus the differential analogues of tables 7 and 8 .

As far as QCD and weak effects are concerned, figures 5 and 6 show rather similar patterns. NLO QCD contributions are dominant everywhere in the phase space, and their 
size increase with the collider energy in a manner which is, in the first approximation, rather independent of the observable or the range considered (however, a closer inspection reveals some minor differences in the shapes of the relative contributions to several observables). In other words, there is no single phase-space region associated with the growth with energy of the relative NLO QCD contribution observed in table 6. At a given collider energy, the NLO QCD $K$ factors are generally not flat, with the exception of $y(t)$ and, to a good extent, of $\Delta y(t \bar{t}, H)$ at $100 \mathrm{TeV}$; the $K$ factors also tend to flatten out at large transverse momenta or invariant masses. The case of NLO weak effects is interesting because they become significant only in certain regions of the phase space (we remind the reader that we are discussing here the analogue of the fully inclusive case of section 3.1, for which at the level of rates weak contributions are smaller than QCD scale uncertainties, as documented by the entries not included in round brackets in table 6). In particular, the histograms that include the NLO weak contributions lie at the lower end of the QCD scale-uncertainty band at large $p_{T}(H), p_{T}(t)$, and (to a somewhat lesser extent) $\Delta y(t \bar{t}, H)$. Weak effects induce therefore a significant distortion of the spectra in those regions, and cannot be neglected. The above regions are rather directly related with those relevant to the boosted scenario; it is therefore consistent with the behaviour of the rates within the cuts of eq. (3.5) shown in table 6 that we observe that the relative importance of NLO weak vs NLO QCD contributions is greater at $13 \mathrm{TeV}$ than at $100 \mathrm{TeV}$.

One has to keep in mind that the impact of the NLO weak effects discussed above can be partly compensated by that of the HBR contributions, since the relative importance of the latter tends to increase (in absolute value) in the same regions where the NLO weak corrections are most significant, at both 13 and $100 \mathrm{TeV}$, as shown by the insets of figures 7 and 8. From these figures, we also see the differential counterpart of table 7: at $13 \mathrm{TeV}$, the interplay of the $g g$ with the $d \bar{d}$ and $u \bar{u}$ channels is involved, while at $100 \mathrm{TeV}$ one is dominated everywhere in the phase space by the $g g$-initiated process.

We conclude this section by presenting in figure 9 the results for our six reference differential distributions obtained by imposing the cuts of eq. (3.5). As expected, the effect of such cuts is that of further enhancing the impact of the NLO weak contributions, which become competitive with the QCD ones, and non-negligible even close to the $p_{T}$ thresholds (compare e.g. the insets of the upper two panels of figures 5 and 9). Note that this conclusion is not modified when the HBR contributions are taken into account, as was already observed for the predictions of the total rates. We finally comment on a few visible features that appear in the differential $p_{T}(t), p_{T}(t \bar{t})$, and $M(t \bar{t} H)$ distributions in the boosted scenario. These are all due to the cuts eq. (3.5), which at the LO result in a sharp threshold in the case of $p_{T}(t \bar{t})$ and $M(t \bar{t} H)$. Such a threshold, which disappears when extra particles may be radiated, is thus a critical point [75] inside the phase space, and therefore a source of instabilities in perturbation theory. We point out that, although hardly visible in figure 9, below threshold the two NLO-accurate results which differ by the presence of the NLO weak effects are not identical; note that, in this region, the latter are solely due to the QCD-type radiation that is responsible for real-emission corrections (i.e. to the term $R_{\mathrm{QCD}, 0} \cdot R_{\mathrm{QCD}, 1}$ that appears in eq. (2.7)). In the case of $p_{T}(t)$, the knee around $400 \mathrm{GeV}$ is due to the fact that the Higgs prefers to stay closer to either the top 
or the antitop than away from both of them (as we have verified by studying the relevant $(\eta, \varphi)$ distances). This results effectively in a non-sharp threshold, which largely disappears when NLO contributions are included; since this threshold is not due to a tight kinematic constraint, the effects are much milder than for $p_{T}(t \bar{t})$ and $M(t \bar{t} H)$.

\section{Conclusions}

In this paper we have presented the first calculation of the $\mathcal{O}\left(\alpha_{S}^{2} \alpha^{2}\right)$ next-to-leading order contribution to $t \bar{t} H$ hadroproduction that includes all weak and QCD effects. The computation is performed in the MADGRAPH5_AMC@NLO framework, and constitutes the first step towards the complete automation, in that framework, of NLO-accurate cross section calculations in theories other than QCD. These are relevant to the perturbative expansion in terms of either a coupling constant different from $\alpha_{S}$ (e.g. the $\mathrm{EW}$ one $\alpha$ ), or simultaneously in more than one coupling constant (e.g. $\alpha_{S}$ and $\alpha$, as in the case studied here). Weak corrections have more interesting physics implications than those of QED origin, which have not been considered in this paper, because of their potential significant impact in phase-space regions characterised by large invariants (as we have documented), and because of the different Higgs couplings they involve. Furthermore, from the point of view of an automated approach which is already capable of computing NLO QCD effects, the case of weak corrections poses a vastly different challenge, and thus offers a better testing context, than that of QED corrections.

We have used $t \bar{t} H$ production as a case study to discuss two issues that will become increasingly frequent in the near future. Firstly, for processes that feature several classes of amplitudes, which differ by the coupling-constant combination they factorise, the intuitive classification of the two dominant next-to-leading order contributions as QCD and EW corrections may become a source of confusion, since in general at the level of cross sections these two kind of corrections mix. Therefore, it is best avoided; we have suggested an alternative terminology, which can be applied to arbitrary processes and perturbative series. Note that this is no longer an academic matter, in view of the fact that automated codes will soon be able to evaluate all contributions to Born and NLO cross sections, regardless of their hierarchy in terms of coupling constants. Secondly, weak contributions due to the emission of potentially resolvable massive EW vector bosons need to be taken into account, at least when one is not able to discard them in the context of a fully realistic analysis at the level of final states. We have shown that, in the case of $t \bar{t} H$ inclusive production, these processes may in fact not be entirely negligible in precision phenomenology studies.

We have compared the $\mathcal{O}\left(\alpha_{S}^{2} \alpha^{2}\right)$ predictions with those of $\mathcal{O}\left(\alpha_{S}^{3} \alpha\right)$, which constitute the dominant (in terms of coupling hierarchy) contribution to NLO effects. We have found that such a hierarchy, established a priori on the basis of the coupling-constant behaviour, is amply respected at the level of fully-inclusive cross sections, for which the scale uncertainty of the latter contribution is significantly larger than the whole $\mathcal{O}\left(\alpha_{S}^{2} \alpha^{2}\right)$ result. This picture does change, however, when one emphasises the role of phase-space regions characterised by some large scale (typically related to a high- $p_{T}$ configuration), which can be done by either looking directly at the relevant kinematics, or at the inclusive level by applying 

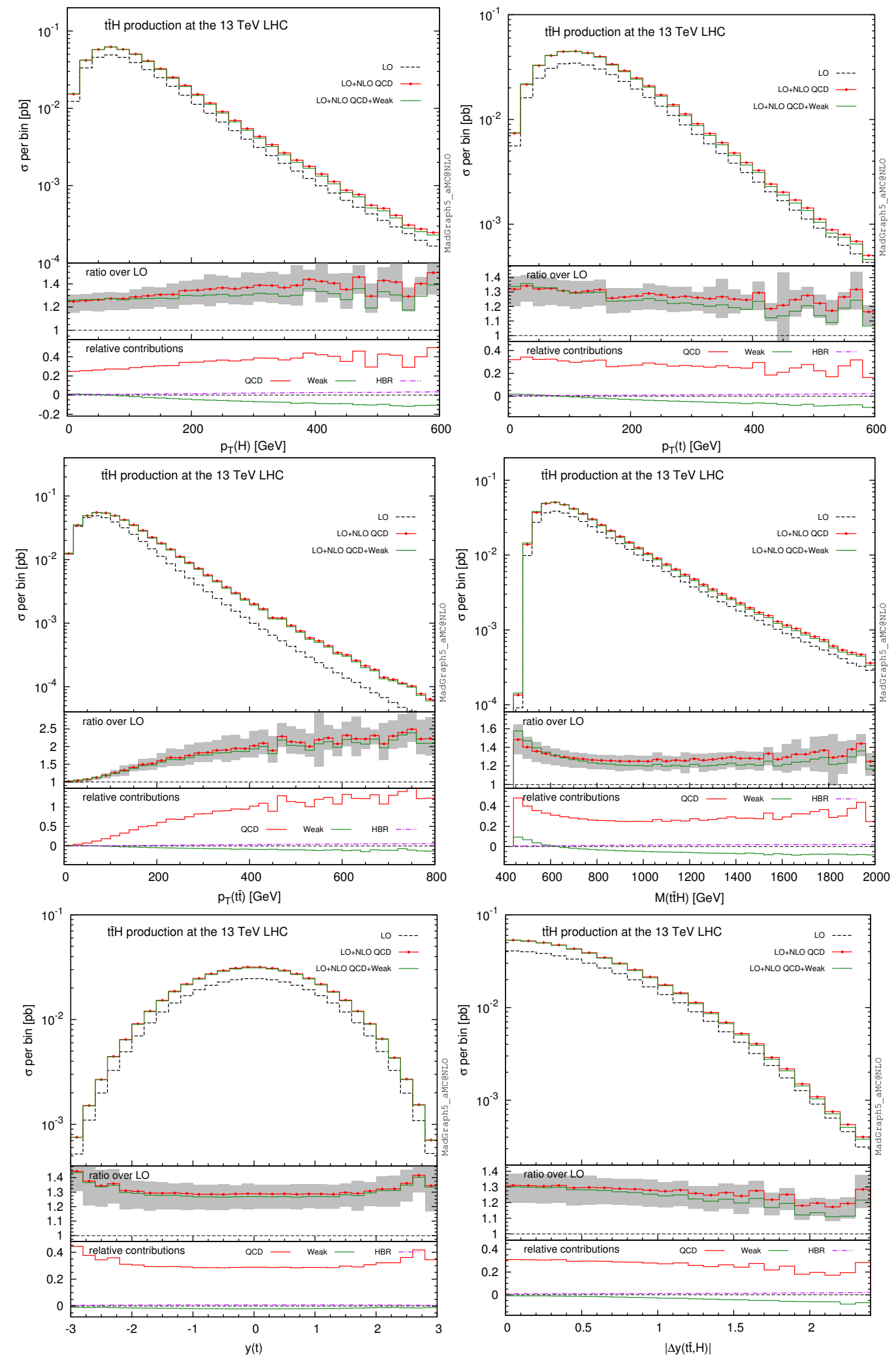

Figure 5. LO- and NLO-accurate results at $13 \mathrm{TeV}$. 

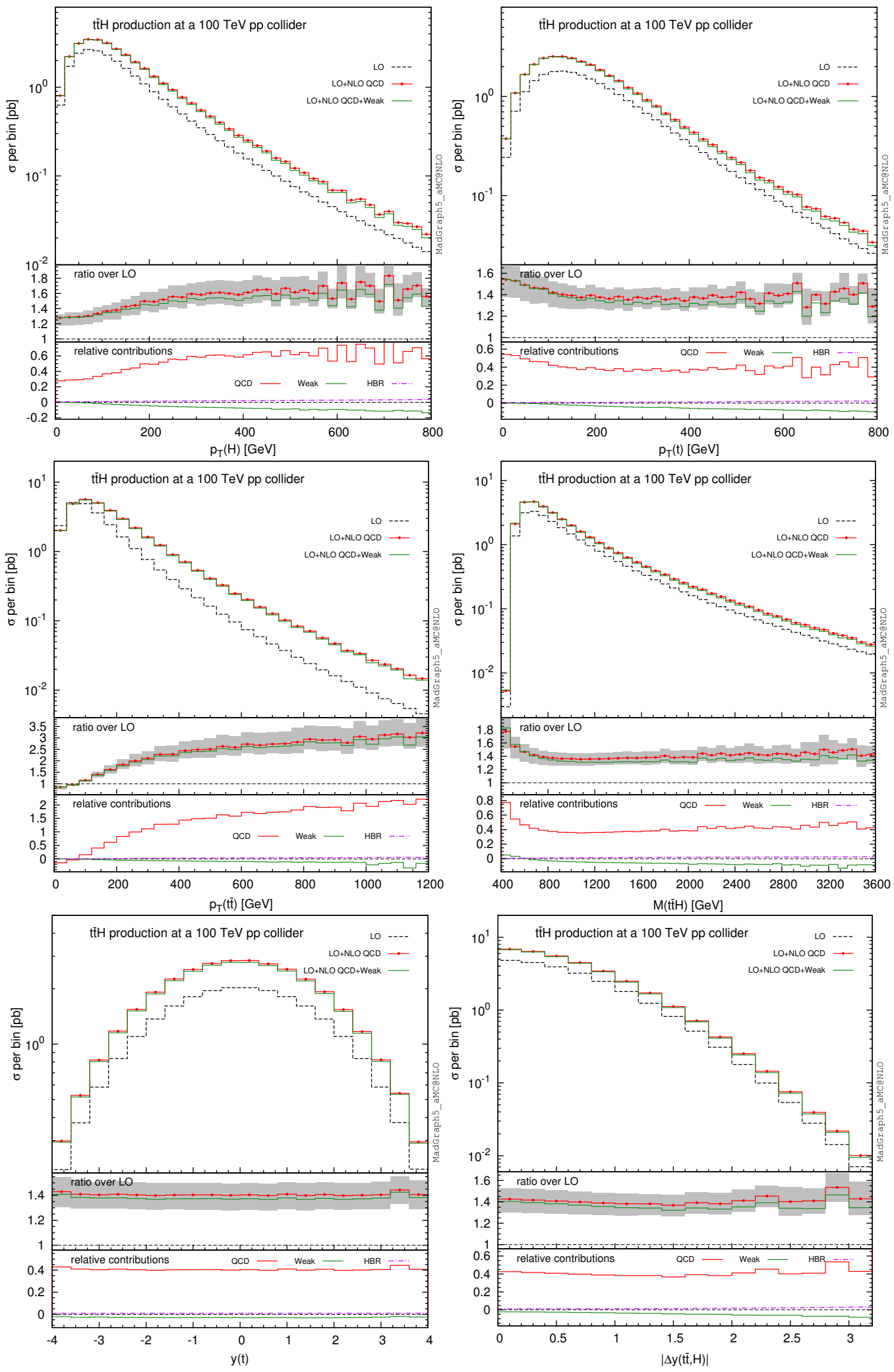

Figure 6. LO- and NLO-accurate results at $100 \mathrm{TeV}$. 

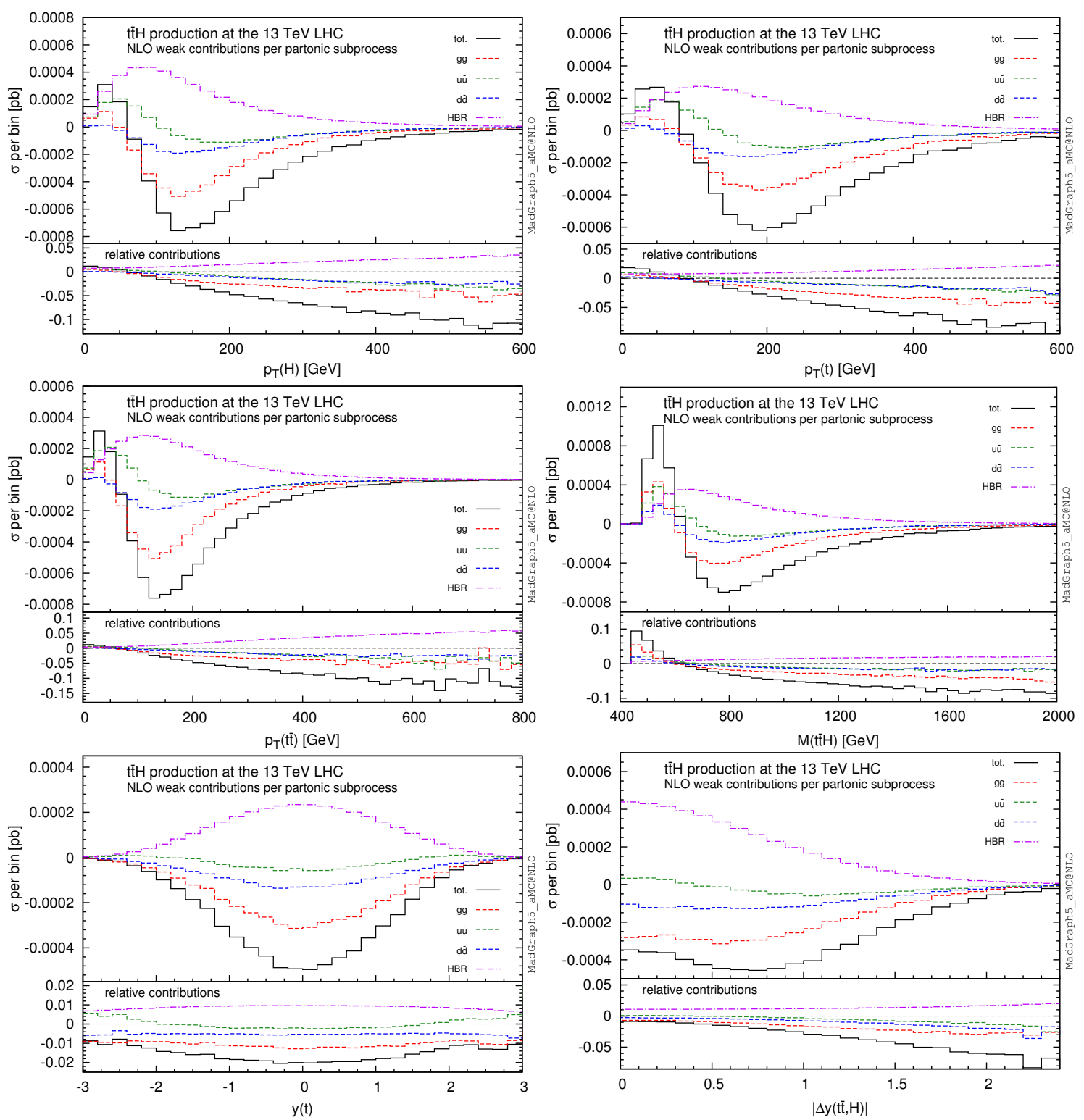

Figure 7. Individual contributions to the NLO weak cross section, at $13 \mathrm{TeV}$.

suitable cuts; both options have been considered here. The main conclusion is that, in these regions, effects of weak origin play an important role, and that $\mathcal{O}\left(\alpha_{S}^{2} \alpha^{2}\right)$ results may be numerically of the same order as the $\mathcal{O}\left(\alpha_{S}^{3} \alpha\right)$ ones. Therefore, $t \bar{t} H$ production appears to follow the same pattern as other processes, where Sudakov logarithms can induce significant distortions of spectra. This implies that the computation of weak contributions is a necessary ingredient for precision phenomenology at large transverse momenta. 

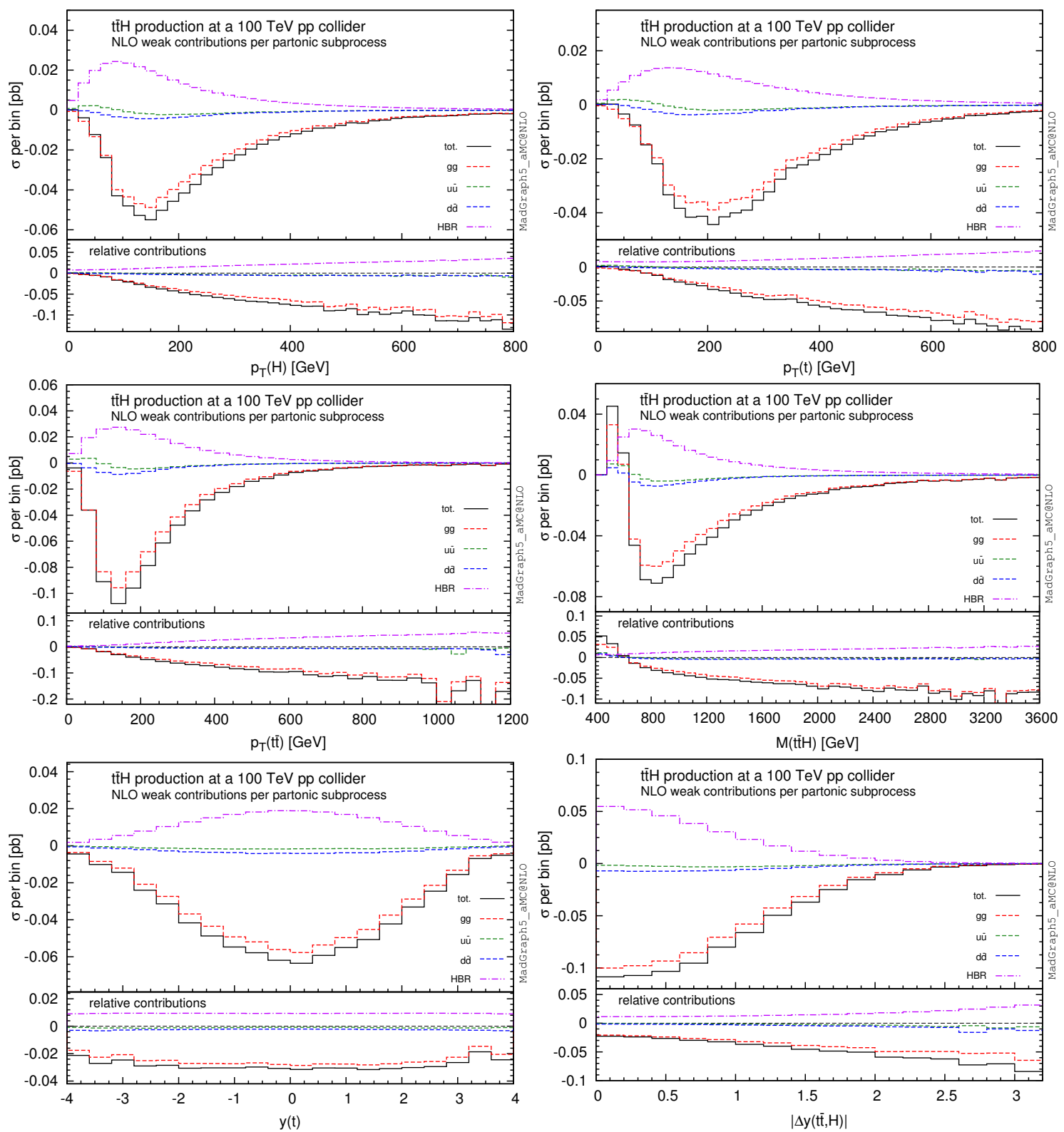

Figure 8. Individual contributions to the NLO weak cross section, at $100 \mathrm{TeV}$.

\section{Acknowledgments}

We thank Fabio Maltoni and Michelangelo Mangano for having encouraged us to pursue the present project. This work is supported in part (DP) by, and performed in the framework of, the ERC grant 291377 "LHCtheory: theoretical predictions and analyses of LHC physics: advancing the precision frontier". The work of VH is supported by the SNF with grant PBELP2 146525. The work of MZ is supported by the Research Executive Agency (REA) of the European Union under the Grant Agreement number PITN-GA2010-264564 (LHCPhenoNet), and by the ILP LABEX (ANR-10-LABX-63), in turn sup- 

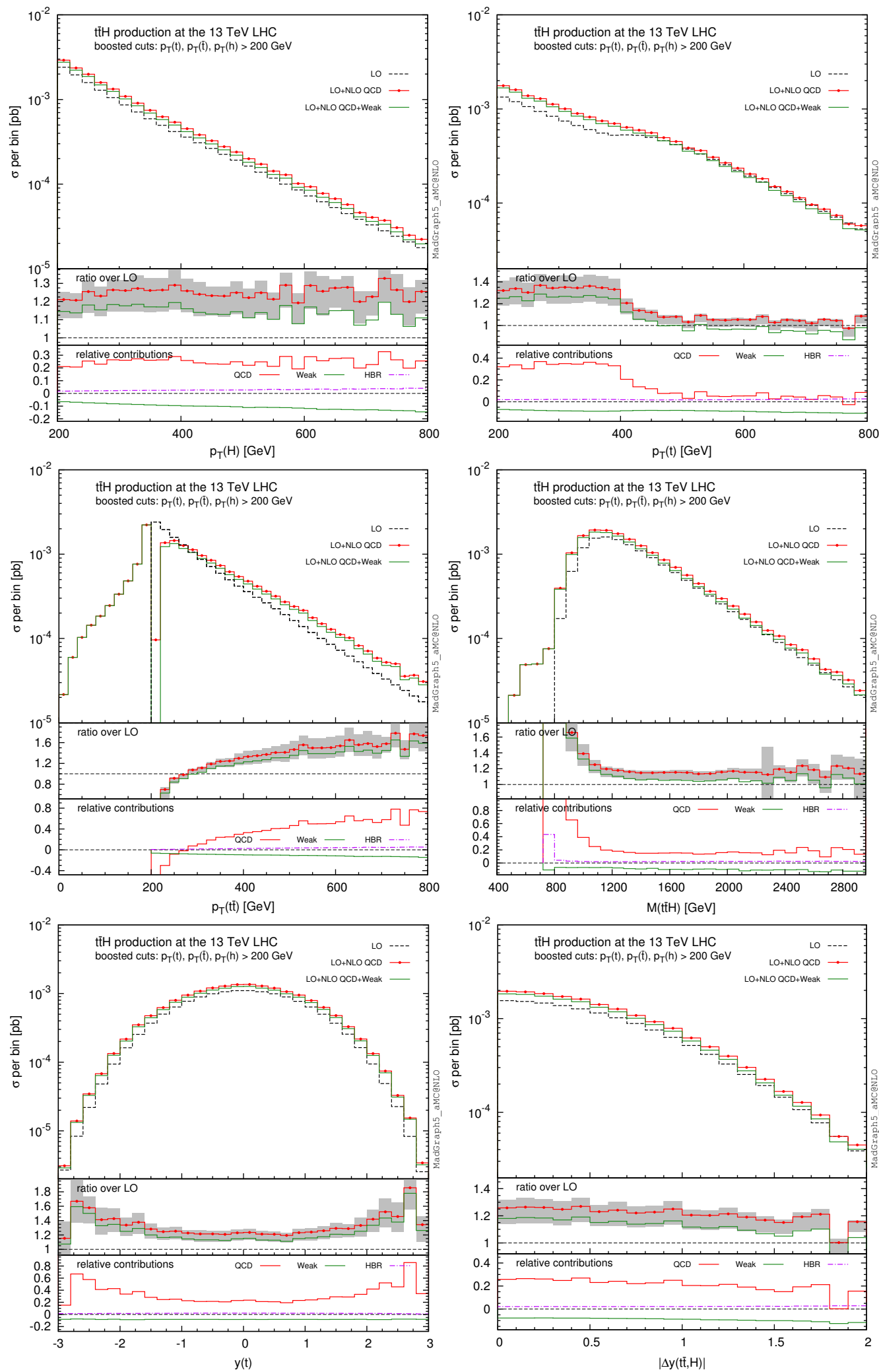

Figure 9. LO- and NLO-accurate results at $13 \mathrm{TeV}$, in the boosted scenario. 
ported by French state funds managed by the ANR within the "Investissements d'Avenir" programme under reference ANR-11-IDEX-0004-02.

Open Access. This article is distributed under the terms of the Creative Commons Attribution License (CC-BY 4.0), which permits any use, distribution and reproduction in any medium, provided the original author(s) and source are credited.

\section{References}

[1] ATLAS collaboration, Observation of a new particle in the search for the standard model Higgs boson with the ATLAS detector at the LHC, Phys. Lett. B 716 (2012) 1 [arXiv: 1207.7214] [INSPIRE].

[2] CMS collaboration, Observation of a new boson at a mass of $125 \mathrm{GeV}$ with the CMS experiment at the LHC, Phys. Lett. B $\mathbf{7 1 6}$ (2012) 30 [arXiv:1207.7235] [INSPIRE].

[3] F. Englert and R. Brout, Broken symmetry and the mass of gauge vector mesons, Phys. Rev. Lett. 13 (1964) 321 [INSPIRE].

[4] P.W. Higgs, Broken symmetries, massless particles and gauge fields, Phys. Lett. 12 (1964) 132 [INSPIRE].

[5] P.W. Higgs, Broken symmetries and the masses of gauge bosons, Phys. Rev. Lett. 13 (1964) 508 [INSPIRE].

[6] CMS collaboration, Study of the mass and spin-parity of the Higgs boson candidate via its decays to Z boson pairs, Phys. Rev. Lett. 110 (2013) 081803 [arXiv:1212.6639] [INSPIRE].

[7] ATLAS collaboration, Evidence for the spin-0 nature of the Higgs boson using ATLAS data, Phys. Lett. B 726 (2013) 120 [arXiv:1307.1432] [InSPIRE].

[8] ATLAS collaboration, Combined coupling measurements of the Higgs-like boson with the ATLAS detector using up to $25 \mathrm{fb}^{-1}$ of proton-proton collision data, ATLAS-CONF-2013-034, CERN, Geneva Switzerland (2013).

[9] CMS collaboration, Observation of a new boson with mass near $125 \mathrm{GeV}$ in pp collisions at $\sqrt{s}=7$ and $8 \mathrm{TeV}$, JHEP 06 (2013) 081 [arXiv:1303.4571] [INSPIRE].

[10] ATLAS collaboration, Search for $t t H$ production in the $H \rightarrow$ yy channel at $\sqrt{s}=8 \mathrm{TeV}$ with the ATLAS detector, ATLAS-CONF-2013-080, CERN, Geneva Switzerland (2013).

[11] ATLAS collaboration, Search for the standard model Higgs boson produced in association with top quarks in proton-proton collisions at $\sqrt{s}=7 \mathrm{TeV}$ using the ATLAS detector, ATLAS-CONF-2012-135, CERN, Geneva Switzerland (2012).

[12] CMS collaboration, Search for the standard model Higgs boson produced in association with a top-quark pair in pp collisions at the LHC, JHEP 05 (2013) 145 [arXiv:1303.0763] [INSPIRE].

[13] CMS collaboration, Search for the standard model Higgs boson produced in association with top quarks in multilepton final states, CMS-PAS-HIG-13-020, CERN, Geneva Switzerland (2013).

[14] CMS collaboration, Search for Higgs boson production in association with a top-quark pair and decaying to bottom quarks or tau leptons, CMS-PAS-HIG-13-019, CERN, Geneva Switzerland (2013). 
[15] CMS collaboration, Search for ttH production in events where $H$ decays to photons at 8 TeV collisions, CMS-PAS-HIG-13-015, CERN, Geneva Switzerland (2013).

[16] CMS collaboration, Search for Higgs boson production in association with top quark pairs in pp collisions, CMS-PAS-HIG-12-025, CERN, Geneva Switzerland (2012).

[17] W. Beenakker et al., Higgs radiation off top quarks at the Tevatron and the LHC, Phys. Rev. Lett. 87 (2001) 201805 [hep-ph/0107081] [InSPIRE].

[18] W. Beenakker et al., NLO QCD corrections to $t \bar{t} H$ production in hadron collisions, Nucl. Phys. B 653 (2003) 151 [hep-ph/0211352] [INSPIRE].

[19] S. Dawson, L.H. Orr, L. Reina and D. Wackeroth, Associated top quark Higgs boson production at the LHC, Phys. Rev. D 67 (2003) 071503 [hep-ph/0211438] [INSPIRE].

[20] S. Dawson, C. Jackson, L.H. Orr, L. Reina and D. Wackeroth, Associated Higgs production with top quarks at the Large Hadron Collider: NLO QCD corrections, Phys. Rev. D 68 (2003) 034022 [hep-ph/0305087] [INSPIRE].

[21] R. Frederix et al., Scalar and pseudoscalar Higgs production in association with a top-antitop pair, Phys. Lett. B 701 (2011) 427 [arXiv:1104.5613] [INSPIRE].

[22] M.V. Garzelli, A. Kardos, C.G. Papadopoulos and Z. Trócsányi, Standard model Higgs boson production in association with a top anti-top pair at NLO with parton showering, Europhys. Lett. 96 (2011) 11001 [arXiv:1108.0387] [INSPIRE].

[23] A. Bredenstein, A. Denner, S. Dittmaier and S. Pozzorini, NLO QCD corrections to $p p \rightarrow t \bar{t} b \bar{b}+X$ at the LHC, Phys. Rev. Lett. 103 (2009) 012002 [arXiv:0905.0110] [INSPIRE].

[24] G. Bevilacqua, M. Czakon, C.G. Papadopoulos, R. Pittau and M. Worek, Assault on the NLO wishlist: $p p \rightarrow t \bar{t} b \bar{b}, J H E P 09$ (2009) 109 [arXiv:0907.4723] [INSPIRE].

[25] A. Bredenstein, A. Denner, S. Dittmaier and S. Pozzorini, NLO QCD corrections to top anti-top bottom anti-bottom production at the LHC: 2 . Full hadronic results, JHEP 03 (2010) 021 [arXiv:1001.4006] [INSPIRE].

[26] A. Kardos and Z. Trócsányi, Hadroproduction of $t \bar{t}$ pair with a b $\bar{b}$ pair using PowHel, J. Phys. G 41 (2014) 075005 [arXiv:1303.6291] [InSPIRE].

[27] F. Cascioli, P. Maierhoefer, N. Moretti, S. Pozzorini and F. Siegert, NLO matching for ttbb production with massive b-quarks, arXiv:1309.5912 [INSPIRE].

[28] A. Djouadi and P. Gambino, Leading electroweak correction to Higgs boson production at proton colliders, Phys. Rev. Lett. 73 (1994) 2528 [hep-ph/9406432] [INSPIRE].

[29] U. Aglietti, R. Bonciani, G. Degrassi and A. Vicini, Two loop light fermion contribution to Higgs production and decays, Phys. Lett. B 595 (2004) 432 [hep-ph/0404071] [InSPIRE].

[30] G. Degrassi and F. Maltoni, Two-loop electroweak corrections to Higgs production at hadron colliders, Phys. Lett. B 600 (2004) 255 [hep-ph/0407249] [InSPIRE].

[31] S. Actis, G. Passarino, C. Sturm and S. Uccirati, NLO electroweak corrections to Higgs boson production at hadron colliders, Phys. Lett. B 670 (2008) 12 [arXiv:0809.1301] [INSPIRE].

[32] M. Ciccolini, A. Denner and S. Dittmaier, Electroweak and QCD corrections to Higgs production via vector-boson fusion at the LHC, Phys. Rev. D 77 (2008) 013002 [arXiv:0710.4749] [INSPIRE]. 
[33] M. Ciccolini, A. Denner and S. Dittmaier, Strong and electroweak corrections to the production of Higgs +2 jets via weak interactions at the LHC,

Phys. Rev. Lett. 99 (2007) 161803 [arXiv:0707.0381] [INSPIRE].

[34] M.L. Ciccolini, S. Dittmaier and M. Krämer, Electroweak radiative corrections to associated $W H$ and $Z H$ production at hadron colliders, Phys. Rev. D 68 (2003) 073003 [hep-ph/0306234] [INSPIRE].

[35] W. Beenakker et al., Electroweak one loop contributions to top pair production in hadron colliders, Nucl. Phys. B 411 (1994) 343 [INSPIRE].

[36] W. Bernreuther, M. Fuecker and Z.G. Si, Mixed QCD and weak corrections to top quark pair production at hadron colliders, Phys. Lett. B 633 (2006) 54 [hep-ph/0508091] [INSPIRE].

[37] J.H. Kuhn, A. Scharf and P. Uwer, Electroweak corrections to top-quark pair production in quark-antiquark annihilation, Eur. Phys. J. C 45 (2006) 139 [hep-ph/0508092] [INSPIRE].

[38] W. Bernreuther, M. Fuecker and Z.-G. Si, Weak interaction corrections to hadronic top quark pair production, Phys. Rev. D 74 (2006) 113005 [hep-ph/0610334] [inSPIRE].

[39] J.H. Kuhn, A. Scharf and P. Uwer, Electroweak effects in top-quark pair production at hadron colliders, Eur. Phys. J. C 51 (2007) 37 [hep-ph/0610335] [InSPIRE].

[40] W. Hollik and M. Kollar, NLO QED contributions to top-pair production at hadron collider, Phys. Rev. D 77 (2008) 014008 [arXiv:0708.1697] [InSPIRE].

[41] W. Bernreuther and Z.-G. Si, Distributions and correlations for top quark pair production and decay at the Tevatron and LHC., Nucl. Phys. B 837 (2010) 90 [arXiv:1003.3926] [INSPIRE].

[42] W. Hollik and D. Pagani, The electroweak contribution to the top quark forward-backward asymmetry at the Tevatron, Phys. Rev. D 84 (2011) 093003 [arXiv:1107.2606] [InSPIRE].

[43] J.H. Kuhn and G. Rodrigo, Charge asymmetries of top quarks at hadron colliders revisited, JHEP 01 (2012) 063 [arXiv:1109.6830] [INSPIRE].

[44] J. Alwall et al., The automated computation of tree-level and next-to-leading order differential cross sections and their matching to parton shower simulations, JHEP 07 (2014) 079 [arXiv:1405.0301] [INSPIRE].

[45] P. Ciafaloni and D. Comelli, Sudakov enhancement of electroweak corrections, Phys. Lett. B 446 (1999) 278 [hep-ph/9809321] [INSPIRE].

[46] M. Ciafaloni, P. Ciafaloni and D. Comelli, Bloch-Nordsieck violating electroweak corrections to inclusive TeV scale hard processes, Phys. Rev. Lett. 84 (2000) 4810 [hep-ph/0001142] [INSPIRE].

[47] A. Denner and S. Pozzorini, One loop leading logarithms in electroweak radiative corrections. 1. Results, Eur. Phys. J. C 18 (2001) 461 [hep-ph/0010201] [InSPIRE].

[48] A. Denner and S. Pozzorini, One loop leading logarithms in electroweak radiative corrections. 2. Factorization of collinear singularities, Eur. Phys. J. C 21 (2001) 63 [hep-ph/0104127] [INSPIRE].

[49] S. Dittmaier, . Kramer, Michael and M. Spira, Higgs radiation off bottom quarks at the Tevatron and the CERN LHC, Phys. Rev. D 70 (2004) 074010 [hep-ph/0309204] [INSPIRE].

[50] V. Hirschi et al., Automation of one-loop QCD corrections, JHEP 05 (2011) 044 [arXiv:1103.0621] [INSPIRE]. 
[51] C. Degrande et al., UFO - the Universal FeynRules Output, Comput. Phys. Commun. 183 (2012) 1201 [arXiv:1108.2040] [INSPIRE].

[52] G. Ossola, C.G. Papadopoulos and R. Pittau, On the rational terms of the one-loop amplitudes, JHEP 05 (2008) 004 [arXiv:0802.1876] [INSPIRE].

[53] S. Dittmaier and. Kramer, Michael, Electroweak radiative corrections to $W$ boson production at hadron colliders, Phys. Rev. D 65 (2002) 073007 [hep-ph/0109062] [INSPIRE].

[54] A. Denner, Techniques for calculation of electroweak radiative corrections at the one loop level and results for $W$ physics at LEP-200, Fortsch. Phys. 41 (1993) 307 [arXiv:0709.1075] [INSPIRE].

[55] M.V. Garzelli, I. Malamos and R. Pittau, Feynman rules for the rational part of the electroweak 1-loop amplitudes, JHEP 01 (2010) 040 [Erratum ibid. 10 (2010) 097] [arXiv:0910.3130] [INSPIRE].

[56] M.V. Garzelli, I. Malamos and R. Pittau, Feynman rules for the rational part of the electroweak 1-loop amplitudes in the $R_{x} i$ gauge and in the unitary gauge, JHEP 01 (2011) 029 [arXiv: 1009.4302] [INSPIRE].

[57] H.-S. Shao, Y.-J. Zhang and K.-T. Chao, Feynman rules for the rational part of the standard model one-loop amplitudes in the 't Hooft-Veltman $\gamma_{5}$ scheme, JHEP 09 (2011) 048 [arXiv:1106.5030] [INSPIRE].

[58] T. Hahn, Generating Feynman diagrams and amplitudes with FeynArts 3, Comput. Phys. Commun. 140 (2001) 418 [hep-ph/0012260] [INSPIRE].

[59] S. Agrawal, T. Hahn and E. Mirabella, Form Calc 7, J. Phys. Conf. Ser. 368 (2012) 012054 [arXiv: 1112.0124] [INSPIRE].

[60] T. Hahn and M. Pérez-Victoria, Automatized one loop calculations in four-dimensions and D-dimensions, Comput. Phys. Commun. 118 (1999) 153 [hep-ph/9807565] [INSPIRE].

[61] G. Altarelli and G. Parisi, Asymptotic freedom in parton language, Nucl. Phys. B 126 (1977) 298 [InSPIRE].

[62] S. Frixione, Z. Kunszt and A. Signer, Three jet cross-sections to next-to-leading order, Nucl. Phys. B 467 (1996) 399 [hep-ph/9512328] [INSPIRE].

[63] S. Frixione, A general approach to jet cross-sections in QCD, Nucl. Phys. B 507 (1997) 295 [hep-ph/9706545] [INSPIRE].

[64] R. Frederix, S. Frixione, F. Maltoni and T. Stelzer, Automation of next-to-leading order computations in QCD: the FKS subtraction, JHEP 10 (2009) 003 [arXiv:0908.4272] [INSPIRE].

[65] G. Ossola, C.G. Papadopoulos and R. Pittau, Reducing full one-loop amplitudes to scalar integrals at the integrand level, Nucl. Phys. B 763 (2007) 147 [hep-ph/0609007] [INSPIRE].

[66] G. Ossola, C.G. Papadopoulos and R. Pittau, CutTools: a program implementing the OPP reduction method to compute one-loop amplitudes, JHEP 03 (2008) 042 [arXiv:0711.3596] [INSPIRE].

[67] F. Cascioli, P. Maierhofer and S. Pozzorini, Scattering amplitudes with open loops, Phys. Rev. Lett. 108 (2012) 111601 [arXiv:1111.5206] [INSPIRE].

[68] A.D. Martin, W.J. Stirling, R.S. Thorne and G. Watt, Parton distributions for the LHC, Eur. Phys. J. C 63 (2009) 189 [arXiv:0901.0002] [InSPIRE]. 
[69] F. Jegerlehner, The effective fine structure constant at TESLA energies, hep-ph/0105283 [INSPIRE].

[70] R. Frederix et al., Four-lepton production at hadron colliders: aMC@NLO predictions with theoretical uncertainties, JHEP 02 (2012) 099 [arXiv:1110.4738] [INSPIRE].

[71] Particle Data Group collaboration, J. Beringer et al., Review of particle physics (RPP), Phys. Rev. D 86 (2012) 010001 [InSPIRE].

[72] J.M. Butterworth, A.R. Davison, M. Rubin and G.P. Salam, Jet substructure as a new Higgs search channel at the LHC, Phys. Rev. Lett. 100 (2008) 242001 [arXiv:0802.2470] [INSPIRE].

[73] T. Plehn, G.P. Salam and M. Spannowsky, Fat jets for a light Higgs, Phys. Rev. Lett. 104 (2010) 111801 [arXiv:0910.5472] [INSPIRE].

[74] M.R. Buckley, T. Plehn, T. Schell and M. Takeuchi, Buckets of Higgs and tops, JHEP 02 (2014) 130 [arXiv:1310.6034] [INSPIRE].

[75] S. Catani and B.R. Webber, Infrared safe but infinite: soft gluon divergences inside the physical region, JHEP 10 (1997) 005 [hep-ph/9710333] [INSPIRE]. 